\title{
Solutions Holomorphes et Singulières d'Équations aux Dérivées Partielles Singulières Non Linéaires
}

\author{
par \\ Raymond GÉrARD* et Hidetoshi TAHARA**
}

\section{Introduction}

Dans l'article [5], nous avons étudié les solutions holomorphes et singulières d'équations aux dérivées partielles singulières non linéaires du premier ordre du type Briot-Bouquet. L'objet du présent travail est d'étendre les résultats de [5] aux équations d'ordre quelconque. Le cas où l'équation est linéaire a été étudié par Tahara [11] et [12].

\section{Notations :}

$\boldsymbol{N}=\{0,1,2, \cdots\}$, ensemble des entiers naturels ;

$N^{*}=\boldsymbol{N} \backslash\{0\} ;$ $x=\left(x_{1}, \cdots, x_{n}\right) \in \boldsymbol{C}^{n}, t \in \boldsymbol{C}$.

Pour tout $\alpha=\left(\alpha_{1}, \cdots, \alpha_{n}\right) \in \boldsymbol{N}^{n}$ nous noterons

$$
|\alpha|=\alpha_{1}+\cdots+\alpha_{n} \text { et }\left(\frac{\partial}{\partial x}\right)^{\alpha}=\left(\frac{\partial}{\partial x_{1}}\right)^{\alpha_{1}} \cdots\left(\frac{\partial}{\partial x_{n}}\right)^{a_{n}} \text {. }
$$

Définissons :

$$
\begin{aligned}
& I_{m}=\left\{(j, \alpha) \in \boldsymbol{N} \times \boldsymbol{N}^{n} ; j+|\alpha| \leqq m \text { et } j<m\right\}, \\
& \delta=\text { le cardinal de } I_{m}, \\
& Z=\left\{Z_{j, a}\right\}(j, \alpha) \in I_{m} \in \boldsymbol{C}^{o} .
\end{aligned}
$$

Soit $\Delta$ un polydisque centré à l'origine de $\boldsymbol{C}_{t} \times \boldsymbol{C}_{x}^{n} \times \boldsymbol{C}_{Z}^{\hat{o}}$; notons

$$
\Delta_{0}=\Delta \cap\{t=0, Z=0\} \text {. }
$$

Soit $F(t, x, Z)$ une fonction de $(t, x, Z)$ définie dans le polydisque $\Delta$ qui satisfait aux hypothèses suivantes :

$$
\left(\mathrm{A}_{1}\right) \quad F(t, x, Z) \text { est holomorphe dans } \Delta \text {; }
$$

Communiqué par T. Kawai, février le 28, 1992.

1991 Mathematics Subject Classification : 35C10.

* Institut de Recherche Mathématique Alsacien, Université Louis Pasteur, 10 rue du Général Zimmer, 67084 Strasbourg, France.

* Department of Mathematics, Sophia University, Kioicho, Chiyoda-ku, 102 Tokyo, Japan. 
( $\left.\mathrm{A}_{2}\right) \quad F(0, x, 0) \equiv 0$ dans $\Delta_{0}$;

$\left(\mathrm{A}_{3}\right) \frac{\partial F}{\partial Z_{j, \alpha}}(0, x, 0) \equiv 0$ dans $\Delta_{0}$ si $|\alpha|>0$.

Introduisons également :

$-\mathscr{R}(\boldsymbol{C} \backslash\{0\})$ le revêtement universel de $\boldsymbol{C} \backslash\{0\}$;

$-S_{\theta}=\{t \in \mathscr{R}(\boldsymbol{C} \backslash\{0\}) ;|\arg t|<\theta\} \quad$ un secteur dans $\mathscr{R}(\boldsymbol{C} \backslash\{0\}) ;$

$-S(\varepsilon(s))=\{t \in \mathscr{R}(C \backslash\{0\}) ; 0<|t|<\varepsilon(\arg t)\}$ où $\varepsilon(s)$ est une fonction définie sur

$\boldsymbol{R}_{s}$ continue et strictement positive;

$-D_{r}=\left\{x \in C^{n} ;\left|x_{\imath}\right| \leqq r, i=1, \cdots, n\right\}$;

$-C\{x\}$ l'anneau des germes de fonctions holomorphes à l'origine de $\boldsymbol{C}_{x}^{n}$;

$-C_{r}\{x\}$ le sous anneau de $C\{x\}$ des fonctions holomorphes dans $D_{r}$;

$\widetilde{\mathcal{O}}_{+}$l'ensemble des fonctions $u(t, x)$ vérifiant les conditions suivantes:

i) il existe une fonction $\varepsilon(s)$ définie sur $\boldsymbol{R}_{s}$ continue et strictement positive et un nombre réel strictement positif $r$ tels que $u(t, x)$ soit holomorphe dans $S(\varepsilon(s)) \times D_{r}$

ii) il existe un nombre réel $a>0$ tel que pour tout $\theta>0$ et toute partie compacte $K$ de $D_{r}$

$$
\max _{x \in K}|u(t, x)|=O\left(|t|^{a}\right)
$$

lorsque $t$ tend vers zéro dans $S_{\theta}$.

Le problème. Déterminer toutes les solutions de l'équation aux dérivées partielles

$$
\left(t \frac{\partial}{\partial t}\right)^{m} u=F\left(t, x,\left\{\left(t \frac{\partial}{\partial t}\right)^{j}\left(\frac{\partial}{\partial x}\right)^{a} u\right\}_{(\jmath, \alpha) \in I_{m}}\right)
$$

qui appartiennent $\grave{a} \widetilde{\mathcal{O}}_{+}$.

Désignons par $\rho_{1}(x), \cdots, \rho_{m}(x)$ les fonctions de $x$ racines du polynôme

$$
C(\rho ; x)=\rho^{m}-\sum_{j<m}\left(\frac{\partial F}{\partial Z_{j, 0}}(0, x, 0)\right) \rho^{j} .
$$

Ces fonctions seront appelées les fonctions caractéristiques de l'équation (E) et $C(\rho ; x)$ le polynôme caractéristique de $(\mathrm{E})$.

Notons

$$
\begin{aligned}
& J_{+}=\left\{i \in\{1, \cdots, m\} ; \operatorname{Re} \rho_{i}(0)>0\right\}, \\
& \mu=\text { le cardinal de } J_{+} .
\end{aligned}
$$

Lorsque $\mu=0$ cette condition signifie donc que $\operatorname{Re} \rho_{i}(0) \leqq 0$ pour tout $i=1, \cdots, m$. Lorsque $\mu \geqq 1$ quitte à changer la numérotation nous pouvons supposer que:

$$
\begin{cases}\operatorname{Re} \rho_{\imath}(0)>0 & \text { pour } 1 \leqq i \leqq \mu, \\ \operatorname{Re} \rho_{i}(0) \leqq 0 & \text { pour } \mu+1 \leqq i \leqq m\end{cases}
$$


Sous la condition de Poincaré (cf. [5], [6]) nous pouvons donner une réponse au problème posé ci-dessus.

\section{Théorème Principal.}

( I ) (Solutions holomorphes). Si $\rho_{i}(0) \notin N^{*}$ pour tout $i=1, \cdots, m$, l'équation (E) a une solution unique $u_{0}(t, x)$ holomorphe au voisinage de l'origine de $\boldsymbol{C}_{t} \times \boldsymbol{C}_{x}^{n}$ vérifiant $u_{0}(0, x) \equiv 0$.

(II) (Solutions singulières). Soit $\mathcal{S}_{+}$l'ensemble des solutions de (E) appartenant à $\widetilde{\mathcal{O}}_{+}$. Alors nous avons:

(II -1) Si $\mu=0, \mathcal{S}_{+}=\left\{u_{0}(t, x)\right\}$.

(II -2) Si $\mu \geqq 1$ et si

i) $\rho_{i}(0) \neq \rho_{j}(0)$ pour $1 \leqq i \neq j \leqq \mu$;

ii) $C(1 ; 0) \neq 0$;

iii) $C\left(i+j_{1} \rho_{1}(0)+\cdots+j_{\mu} \rho_{\mu}(0) ; 0\right) \neq 0$ pour tout $(i, j) \in N \times N^{\mu}$ satisfaisant $\grave{a} i+|j| \geqq 2$;

alors pour tout $\varphi_{1}(x), \cdots, \varphi_{\mu}(x) \in C\{x\}$ l'équation (E) admet une solution $U\left(\varphi_{1}(x)\right.$, $\left.\cdots, \varphi_{\mu}(x)\right)$ appartenant $\grave{a} \widetilde{\mathcal{O}}_{+}$de la forme

$$
\begin{aligned}
& U\left(\varphi_{1}(x), \cdots, \varphi_{\mu}(x)\right)=\sum_{i \geqq 1} u_{i}(x) t^{i} \\
& \quad+\sum_{i+2 m|j| \geq \mid>k+2 m} \varphi_{i, j, k}(x) t^{i+j_{1} \rho_{1}(x)+\cdots+\mu_{\mu} \rho_{\mu}(x)}(\log t)^{k}
\end{aligned}
$$

avec $\varphi_{0, e_{p}, 0}(x)=\varphi_{p}(x), p=1, \cdots, \mu$ où $e_{1}=(1,0, \cdots, 0), \cdots, e_{\mu}=(0, \cdots, 0,1) . \quad$ De plus, nous avons

$$
\mathcal{S}_{+}=\left\{U\left(\varphi_{1}(x), \cdots, \varphi_{\mu}(x)\right) ; \varphi_{p}(x) \in C\{x\}, p=1, \cdots, \mu\right\} .
$$

Remarques. (1) Quand $m=1$, ce théorème nous redonne le résultat de Gérard-Tahara [5].

(2) Lorsque l'équation est linéaire, un résultant plus complet a été donné dans Tahara [11] et [12].

L'exemple suivant explique la différence entre le cas linéaire et le cas non linéaire.

Exemple. Considérons

$$
t \frac{\partial u}{\partial t}=(1+2 \sqrt{-1}) u+a t u \frac{\partial u}{\partial x},
$$

où $(t, x) \in \boldsymbol{C} \times \boldsymbol{C}$ et $a \in \boldsymbol{C}$. Si $a=0$ l'équation (0.3) est linéaire, et si $a \neq 0$ (0.3) est non linéaire. Alors:

(1) Par le théorème principal nous avons

$$
\mathcal{S}_{+}=\{U(\varphi(x)) ; \varphi(x) \in \boldsymbol{C}\{x\}\}
$$

et $U(\varphi(\lambda))$ est de la forme 


$$
U(\varphi(x))=\sum_{k=0}^{\infty} \varphi_{k}(x) t^{1+2 \sqrt{-1}+2 k(1+\sqrt{-1)}},
$$

où les coefficients $\varphi_{k}(x)(k \geqq 0)$ sont déterminés par $\varphi_{0}(x)=\varphi(x)$ et

$$
\varphi_{k}(x)=\frac{a}{k(2+2 \sqrt{-1})} \sum_{i+j=k-1} \varphi_{i}(x)\left(\frac{d \varphi_{j}(x)}{d x}\right), \quad k \geqq 1 .
$$

(2) Si nous prenons $\varphi(x)=x$, la solution $U(\varphi(x))$ se réduit à

$$
u^{*}=x t^{1+2 \sqrt{-1}} \sum_{k=0}^{\infty}\left(\frac{a}{2+2 \sqrt{-1}} t^{2+2 \sqrt{-1}}\right)^{k} .
$$

Le domaine de la convergence de $u^{*}$ est

$$
\begin{cases}\mathscr{R}(\boldsymbol{C} \backslash\{0\}) \times C, & \text { quand } a=0, \\ S(\varepsilon(s)) \times C, & \text { quand } a \neq 0\end{cases}
$$

avec $\varepsilon(s)=(2 \sqrt{2} /|a|)^{1 / 2} e^{s}$.

(3) Soit $\mathcal{S}$ l'ensemble des solutions $u(t, x)$ de (0.3) telles que $u(t, x)$ soit holomorphe dans $S(\varepsilon(s)) \times D_{r}$ pour une fonction $\varepsilon(s)>0$ et un nombre $r>0$. Alors :

$$
\begin{cases}\mathcal{S}=\mathcal{S}_{+}, & \text {quand } a=0, \\ \mathcal{S} \supsetneq \mathcal{S}_{+}, & \text {quand } a \neq 0 .\end{cases}
$$

La preuve est comme ce qui suit. Quand $a=0, \mathcal{S}=\mathcal{S}_{+}$est la conséquence de Tahara [11]. Quand $a \neq 0$, l'équation (0.3) admet une famille de solutions de la forme

$$
\frac{-2-2 \sqrt{-1}}{a} \frac{x+c}{t}, \quad c \in C
$$

qui n'appartiennent pas à $\mathcal{S}_{+}$.

(4) Lorsque $a \neq 0$, il semble difficile de déterminer la structure de $\mathcal{S}$. Nous présentons ceci comme un problème ouvert.

Cet article est présenté de la manière suivante. Dans le $\S 1$ nous donnons les solutions holomorphes de (E). Dans les $\S 2$ et 3 nous construisons une famille de solutions appartenant à $\widetilde{\mathcal{O}}_{+}$. La discussion des paragraphes 1,2 et 3 est basée sur les résultats de [7]. Dans le $\$ 4$ nous rappelons la théorie asymptotique développée dans Tahara [14]. L'utilisation de tous ces résultats nous permet de démontrer le théorème principal dans le $\S 5$.

\section{$\S 1$. Solutions Holomorphes}

Dans cette section, nous rappelons un résultat de [7] sur la convergence des solutions séries formelles d'équations aux dérivées partielles singulières non 
linéaires.

Soient $m \in \boldsymbol{N}^{*}, d \in \boldsymbol{N}^{*}, t=\left(t_{1}, \cdots, t_{d}\right) \in \boldsymbol{C}^{d}$ et $x=\left(x_{1}, \cdots, x_{n}\right) \in \boldsymbol{C}^{n}$ et $a_{i, j}(x)$ $(1 \leqq j \leqq i \leqq d)$ des fonctions holomorphes dans un disque $D_{r}=\left\{x \in \boldsymbol{C}^{n} ;\left|x_{i}\right| \leqq r\right.$, $i=1, \cdots, n\}$ et introduisons le champ de vecteurs

$$
\tau=\sum_{i=1}^{d}\left(\sum_{i=1}^{i} a_{i, j}(x) t_{j}\right) \frac{\partial}{\partial t_{i}} .
$$

Notons :

$$
\begin{aligned}
& I(m)=\left\{(\alpha, \beta, \gamma) \in \boldsymbol{N}^{d} \times \boldsymbol{N}^{d} \times \boldsymbol{N}^{n} ;|\alpha|=|\beta| \leqq m \text { et }|\beta|+|\gamma| \leqq m\right\}, \\
& \delta(m)=\text { le cardinal de } I(m), \\
& Z=\left\{Z_{\alpha, \beta, \gamma}\right\}_{(a, \beta, \gamma) \in I(m)} .
\end{aligned}
$$

Soit $G_{2}(x)\left(t_{1}, \cdots, t_{d}, Z\right)$ une fonction holomorphe de $\left(x, t_{1}, \cdots, t_{d}, Z\right)$ définie dans un polydisque centré à l'origine de $C^{n} \times C^{d} \times C^{\delta(m)}$ ayant la forme suivante:

$$
G_{2}(x)\left(t_{1}, \cdots, t_{d}, Z\right)=\sum_{\mid p_{1+|q| \geq 2}} g_{p, q}(x) t_{1}^{p_{1}} \cdots t_{d}^{p_{d} d} Z^{q}
$$

où

$$
\begin{aligned}
& p=\left(p_{1}, \cdots, p_{d}\right) \in N^{d},|p|=p_{1}+\cdots+p_{d}, \\
& q=\left\{q_{\alpha, \beta, \gamma}\right\}_{(\alpha, \beta, \gamma) \in I(m)} \in N^{\delta(m)}, \\
& |q|=\sum_{(\alpha, \beta, \gamma) \in I(m)} q_{a, \beta, \gamma}, \\
& Z^{q}=\prod_{(\alpha, \beta, \gamma) \in I(m)}\left(Z_{a, \beta, \gamma}\right)^{q_{a}, \beta, \gamma}
\end{aligned}
$$

et pour tout $p, q$ tel que $|p|+|q| \geqq 2$ les fonctions $g_{p, q}(x)$ sont holomorphes dans un même disque centré à l'origine de $\boldsymbol{C}^{n}$.

Pour simplifier la présentation nous écrivons $\nabla^{m} u$ pour

$$
\left\{t^{\alpha}\left(\frac{\partial}{\partial t}\right)^{\beta}\left(\frac{\partial}{\partial x}\right)^{\gamma} u\right\}_{(\alpha, \beta, \gamma) \in I(m)}
$$

où

$$
\begin{gathered}
\alpha=\left(\alpha_{1}, \cdots, \alpha_{d}\right) \in N^{d}, \quad \beta=\left(\beta_{1}, \cdots, \beta_{d}\right) \in N^{d}, \quad \gamma=\left(\gamma_{1}, \cdots, \gamma_{n}\right) \in N^{n}, \\
t^{a}=t_{1}^{\alpha_{1}} \cdots t_{d}^{\alpha}, \quad\left(\frac{\partial}{\partial t}\right)^{\beta}=\left(\frac{\partial}{\partial t_{1}}\right)^{\beta_{1}} \cdots\left(\frac{\partial}{\partial t_{d}}\right)^{\beta_{d}}, \quad\left(\frac{\partial}{\partial x}\right)^{\gamma}=\left(\frac{\partial}{\partial x_{1}}\right)^{\gamma_{1}} \cdots\left(\frac{\partial}{\partial x_{n}}\right)^{\gamma_{n}} .
\end{gathered}
$$

Soient $c_{j}(x)(0 \leqq j \leqq m-1)$ et $b_{i}(x)(1 \leqq i \leqq d)$ des fonctions holomorphes dans $D_{r}$. On peut donc considérer l'équation

$$
\left(\tau^{m}+c_{m-1}(x) \tau^{m-1}+\cdots+c_{0}(x)\right) u=\sum_{i=1}^{d} b_{i}(x) t_{i}+G_{2}(x)\left(t_{1}, \cdots, t_{d}, \nabla^{m} u\right)
$$

qui est un cas particulier de l'équation étudiée dans [7].

Dans cet article, par série formelle nous entendons une série formelle en $t$ de la forme 


$$
\hat{u}(t, x)=\sum_{|p| \geq 1} u_{p}(x) t_{1}^{p_{1}} \cdots t_{d}^{p} d
$$

où les coéfficients $u_{p}(x)$ sont tous holomorphes dans un même polydisque centré à l'origine de $C^{n}$; une solution formelle de $\left(\mathrm{E}_{1}\right)$ est par définition une série formelle de la forme $\hat{u}(t, x)$ qui vérifie formellement $\left(E_{1}\right)$.

Désignons par $\lambda_{1}(x), \cdots, \lambda_{m}(x)$ les racines du polynôme

$$
P(\lambda ; x)=\lambda^{m}+\sum_{j<m} c_{j}(x) \lambda^{j} .
$$

D’après le $\S 3$ de [7] nous avons :

Théorème 1. Si l'origine de $C$ n'appartient pas à l'enveloppe convexe dans $C$ de l'ensemble de points $\left\{a_{1,1}(0), \cdots, a_{d, d}(0)\right\}$, alors:

(1) Toute solution formelle de $\left(\mathrm{E}_{1}\right)$ est convergente au voisinage de l'origine de $\boldsymbol{C}^{d} \times \boldsymbol{C}^{n}$.

(2) $\mathrm{Si}$

$$
\begin{array}{cl}
\sum_{i=1}^{d} p_{i} a_{i, i}(0)-\lambda_{j}(0) \neq 0 & \text { pour tout } \\
\left(p_{1}, \cdots, p_{d}\right) \in N^{d} \backslash\{(0, \cdots, 0)\} & \text { et } \quad j=1, \cdots, m ;
\end{array}
$$

l'équation $\left(\mathrm{E}_{1}\right)$ admet une solution formelle unique qui donne une solution holomorphe unique $u(t, x)$ vérifiant $u(0, x) \equiv 0$.

L'équation (E) introduite dans l'introduction étant un cas particulier d'équations $\left(\mathrm{E}_{1}\right)$ nous avons :

Corollaire 1. Si $\rho_{i}(0) \notin N^{*}$ pour tout $i=1, \cdots, m$, l'équation (E) admet une solution unique $u(t, x)$ holomorphe au voisinage de l'origine de $C \times C^{n}$ vérifiant $u(0, x) \equiv 0$.

Pour d'autres résultats concernant l'existence de solutions holomorphes, voir Gérard [2] et [3] ainsi que Madi-Yoshino [10].

\section{§2. Solutions Singulières: Cas Spécial}

Dans cette section, nous étudions une classe assez restreinte d'équations aux dérivées partielles singulières non linéaires et construisons une famille de solutions singulières appartenant à $\widetilde{O}_{+}$. Cette classe restreinte d'équations a l'advatage dans la recherche des solutions appartenant à $\widetilde{\mathcal{O}}_{+}$de pouvoir être réduite à une équation $\left(E_{1}\right)$ et de permettre l'utilisation du théorème 1 .

Soient $m \in N^{*}, \quad(t, x) \in C \times C^{n}, \quad x=\left(x_{1}, \cdots, x_{n}\right), \quad \alpha=\left(\alpha_{1}, \cdots, \alpha_{n}\right) \in \boldsymbol{N}^{n},|\alpha|=$ $\alpha_{1}+\cdots+\alpha_{n}$ et

$$
\left(t \frac{\partial}{\partial x}\right)^{\alpha}=\left(t \frac{\partial}{\partial x_{1}}\right)^{\alpha} \cdots\left(t \frac{\partial}{\partial x_{n}}\right)^{a_{n}}
$$


Soient $b(x)$ et $c_{\jmath}(x)(0 \leqq j \leqq m-1)$ des fonctions définies et holomorphes dans un polydisque centré à l'origine de $\boldsymbol{C}^{n}$.

Notons

$$
Z=\left\{Z_{j, \alpha}\right\}_{j+|a| \leqq m}, \quad Z_{j, \alpha} \in C
$$

et soit $G_{2}(x)(t, Z)$ une fonction définie et holomorphe dans un polydisque centré à l'origine $(x=0, t=0, Z=0)$ ayant de plus la forme suivante:

$$
G_{2}(x)(t, Z)=\sum_{p+|q| \geqq 2} g_{p, q}(x) t^{p} Z^{q},
$$

où

$$
\begin{aligned}
q & =\left\{q_{j, \alpha}\right\}_{j+|\alpha| \leqq m}, \quad q_{j, a} \in N, \\
|q| & =\sum_{j+|\alpha| \leqq m} q_{j, a}, \\
Z^{q} & =\prod_{j+|\alpha| \leqq m}\left(Z_{j, a}\right)^{q_{j, \alpha}}
\end{aligned}
$$

et $g_{p, q}(x)(p+|q| \geqq 2)$ sont des fonctions holomorphes dans un même polydisque centré à l'origne de $\boldsymbol{C}^{n}$.

Considèrons l'équation aux dérivées partielles

$$
\begin{aligned}
\left(\left(t \frac{\partial}{\partial t}\right)^{m}\right. & \left.+c_{m-1}(x)\left(t \frac{\partial}{\partial t}\right)^{m-1}+\cdots+c_{0}(x)\right) u \\
& =t b(x)+G_{2}(x)\left(t,\left\{\left(t \frac{\partial}{\partial t}\right)^{j}\left(t \frac{\partial}{\partial x}\right)^{\alpha} u\right\}_{j+|\alpha| \leq m}\right)
\end{aligned}
$$

et posons nous le problème de chercher une solution singulière de cette équation.

Désignons par $\rho_{1}(x), \cdots, \rho_{m}(x)$ les exposants caractéristique de cette équation donc les racines du polynôme

$$
C(\rho ; x)=\rho^{m}+\sum_{j<m} c_{j}(x) \rho^{\jmath} .
$$

Nous avons :

Théorème 2. Soit $1 \leqq l \leqq m$. Supposons :

i) $\rho_{1}(x), \cdots, \rho_{l}(x)$ holomorphes au voisinage de $x=0$;

ii) $\operatorname{Re} \rho_{1}(0)>0, \cdots, \operatorname{Re} \rho_{l}(0)>0$;

iii) $C(1 ; 0) \neq 0$;

iv) $C\left(i+j_{1} \rho_{1}(0)+\cdots+j_{l} \rho_{l}(0)+k ; 0\right) \neq 0$ pour tout $(i, j, k) \in \boldsymbol{N} \times \boldsymbol{N}^{l} \times \boldsymbol{N}$ vérifiant $i+|j|+k \geqq 2$.

Alors l'équation $\left(\mathrm{E}_{2}\right)$ a une famille de solutions appartenant $\grave{a} \widetilde{\mathcal{O}}_{+}$de la forme

$$
\sum_{i+|j|+k \geqq 1} \varphi_{i, j, k}(x) t^{i}\left(t^{\rho_{1}(x)}\right)^{j_{1}} \cdots\left(t^{\rho_{l}(x)}\right)^{j_{l}}(t \log t)^{k},
$$

où tous les coéfficients $\varphi_{i, j, k}(x)(i+|j|+k \geqq 1)$ sont holomorphes dans un même disque centré à l'origine de $\boldsymbol{C}^{n}$; de plus toute solution de $\left(\mathrm{E}_{2}\right)$ est déterminée de 
manière unique par la donnée des coéfficients $\left\{\varphi_{0, j, 0}(x) ;|j|=1\right\}$ qui sont arbitraires. Si nous prenous $\varphi_{0,3,0}(x) \equiv 0$ pour tout $j$ tel que $|j|=1$, la solution (2.2) se réduit à l'unique solution holomorphe de l'éqation $\left(\mathrm{E}_{2}\right)$.

Preuve. Pour commencer nous allons chercher une solution "formelle" de la forme

$$
\hat{\imath}(t, x)=w\left(t, t^{\rho_{1}(x)}, \cdots, t^{\rho_{l}(x)}, t \log t, x\right),
$$

où

$$
w\left(t_{0}, t_{1}, \cdots, t_{l}, t_{l+1}, x\right)=\sum_{i+|j|+k \geq 1} \varphi_{\imath, j, k}(x)\left(t_{0}\right)^{i}\left(t_{1}\right)^{j_{1}} \cdots\left(t_{l}\right)^{j l}\left(t_{l+1}\right)^{k}
$$

est série formelle en $t_{0}, t_{1}, \cdots, t_{l}, t_{l+1}$ à coéfficients holomorphes dans un polydisque commun de $C^{n}$ : ensuite nous démontrerons la convergence de cette série.

Posons

$$
t_{0}=t, \quad t_{p}=t^{\rho_{p}(x)}(p=1, \cdots, l) \text { et } t_{l+1}=t \log t .
$$

Nous avons formellement

$$
\begin{gathered}
t \frac{\partial u}{\partial t}=t_{0} \frac{\partial w}{\partial t_{0}}+\sum_{p=1}^{l} \rho_{p}(x) t_{p} \frac{\partial w}{\partial t_{p}}+\left(t_{0}+t_{l+1}\right) \frac{\partial w}{\partial t_{l+1}}, \\
t \frac{\partial u}{\partial x_{q}}=t_{0} \frac{\partial w}{\partial x_{q}}+t_{l+1} \sum_{p=1}^{l} \frac{\partial \rho_{p}(x)}{\partial x_{q}} t_{p} \frac{\partial w}{\partial t_{p}}, \quad q=1, \cdots, n .
\end{gathered}
$$

Donc $\hat{u}(t, x)$ est une solution formelle de $\left(\mathrm{E}_{2}\right)$ si et seulement si $w\left(t_{0}, t_{1}, \cdots, t_{l}\right.$, $\left.t_{l+1}, x\right)$ est une solution formelle de l'équation:

$$
\left(T^{m}+c_{m-1}(x) T^{m-1}+\cdots+c_{0}(x)\right) w=t_{0} b(x)+G_{2}(x)\left(t_{0},\left\{T^{\jmath} X^{\alpha} w\right\}_{\jmath+|a| \leq m}\right),
$$

où $T$ et $X_{1}, \cdots, X_{n}$ sont les champs de vecteurs suivants:

$$
\begin{gathered}
T=t_{0} \frac{\partial}{\partial t_{0}}+\sum_{p=1}^{l} \rho_{p}(x) t_{p} \frac{\partial}{\partial t_{p}}+\left(t_{0}+t_{l+1}\right) \frac{\partial}{\partial t_{l+1}}, \\
X_{q}=t_{0} \frac{\partial}{\partial x_{q}}+t_{l+1} \sum_{p=1}^{l} \frac{\partial \rho_{p}(x)}{\partial x_{q}} t_{p} \frac{\partial}{\partial t_{p}}, \quad q=1, \cdots, n .
\end{gathered}
$$

Nous remarquons que l'équation $\left(E_{2}^{\prime}\right)$ est un cas particulier d'équation $\left(E_{1}\right)$ discutée dans le $\S 1$ et que le champ de vecteurs $T$ satisfait à une condition de Poincaré (0 n'appartient pas à l'enveloppe convexe dans $C$ de l'ensemble de points $\left.\left\{1, \rho_{1}(0), \cdots, \rho_{l}(0)\right\}\right)$. De plus, par les conditions i), ii) et iii) nous voyons qu'il existe un nombre réel $r>0$ tel que:

$$
\begin{cases}\text { 1) } & C(1 ; x) \neq 0 \quad \text { dans } D_{r} ; \\ \text { 2) } & C\left(i+j_{1} \rho_{1}(x)+\cdots+j_{l} \rho_{l}(x)+k ; x\right) \doteq 0 \text { dans } D_{r} \text { pour } \\ & \text { tout }(i, j, k) \in \boldsymbol{N} \times \boldsymbol{N}^{l} \times \boldsymbol{N} \text { satisfaisant à } i+|j|+k \geqq 2 .\end{cases}
$$


Par conséquent pour avoir l'existence de solutions de l'équation $\left(\mathrm{E}_{2}\right)$ de la forme (2.2) appartenant à $\widetilde{\mathcal{O}}_{+}$il est suffisant de prouver :

Proposition 1. Soit $1 \leqq l \leqq m$. Supposons $\rho_{1}(x), \cdots, \rho_{l}(x)$ holomorphes au voisinage de $x=0$, que 0 n'appartient pas à l'enveloppe convexe dans $\boldsymbol{C}$ des points $1, \rho_{1}(0), \cdots, \rho_{l}(0)$ et que les conditions (2.4) sont satisfaites pour un $r>0$. Alors, l'équation ( $\left.\mathrm{E}_{2}^{\prime}\right)$ admet une famille de solutions de la forme

$$
\sum_{i+1, j \mid+k \geqq 1} \varphi_{i, j, k}(x)\left(t_{0}\right)^{i}\left(t_{1}\right)^{j_{1}} \cdots\left(t_{l}\right)^{j l}\left(t_{l+1}\right)^{k},
$$

où tous les coéfficients $\varphi_{i, j, k}(x)(i+||+k \geqq 1$,$) sont déterminés de manière unique$ par les coéfficients $\left\{\varphi_{0, j, 0}(x) ;|j|=1\right\}$ qui eux sont arbitraires.

Démonstration de la proposition 1. Posons formellement

$$
w=\sum_{s=1}^{\infty} w_{s}\left(t_{0}, t_{1}, \cdots, t_{l}, t_{l+1}, x\right)
$$

où pour tout $s \geqq 1$

$$
w_{s}=\sum_{i+|j|+k=8} \varphi_{i, j, k}(x)\left(t_{0}\right)^{i}\left(t_{1}\right)^{j_{1}} \cdots\left(t_{l}\right)^{j l}\left(t_{l+1}\right)^{k} .
$$

L'équation $\left(\mathrm{E}_{2}^{\prime}\right)$ se décompose alors en les équations

$$
\left\{\begin{array}{l}
C(T ; x) w_{1}=b(x) t_{0}, \\
C(T ; x) w_{s}=F_{s}\left(w_{1}, \cdots, w_{s-1}\right), \quad s \geqq 2,
\end{array}\right.
$$

où $F_{s}\left(w_{1}, \cdots, w_{s-1}\right)$ est un polynôme homogène de degré $s$ en $\left(t_{0}, t_{1}, \cdots, t_{l}, t_{l+1}\right)$ à coéfficients holomorphes en $x$ et entierement déterminés par $w_{1}, \cdots, w_{s-1}$. De plus en regardant (2.6) nous voyons

$$
\begin{aligned}
C(T ; x) w_{1}= & \left(C(1 ; x) \varphi_{1,0,0}(x)+\frac{\partial C}{\partial \rho}(1 ; x) \varphi_{0,0,1}(x)\right) t_{0} \\
& +\sum_{p=1}^{l} C\left(\rho_{p}(x) ; x\right) \varphi_{0, e_{p}, 0}(x) t_{p}+C(1 ; x) \varphi_{0,0,1}(x) t_{l+1}
\end{aligned}
$$

où $e_{1}=(1,0, \cdots, 0), \cdots, e_{l}=(0, \cdots, 0,1) \in \boldsymbol{N}^{l}$, et pour $s \geqq 2$

$$
\begin{aligned}
C(T ; x) w_{s}= & \sum_{i+|j|+k=s}\left\{C\left(i+j_{1} \rho_{1}(x)+\cdots+j_{l} \rho_{l}(x)+k ; x\right) \varphi_{i, j, k}(x)\right. \\
& \left.+\sum_{h=1}^{m} C_{h}(i, j, k ; x) \varphi_{i-h, j, k+h}(x)\right\}\left(t_{0}\right)^{i}\left(t_{1}\right)^{j_{1}} \cdots\left(t_{l}\right)^{j_{l}}\left(t_{l+1}\right)^{k}
\end{aligned}
$$

où par convention $\varphi_{i-h, j, k+h}(x) \equiv 0$ pour $i-h<0$ et où

$$
\begin{aligned}
& C_{h}(i, j, k ; x) \\
& \quad=\sum_{q=h}^{m}\left(\begin{array}{l}
q \\
h
\end{array}\right) c_{q}(x)\left(i+j_{1} \rho_{1}(x)+\cdots+j_{l} \rho_{l}(x)+k\right)^{q-h}(k+1)(k+2) \cdots(k+h)
\end{aligned}
$$


et $c_{m}(x) \equiv 1$.

En conséquence, par (2.4), (2.7), (2.8) et le fait que

$$
C\left(\rho_{p}(x) ; x\right) \equiv 0 \quad(p=1, \cdots, l)
$$

nous avons

1) $\varphi_{1,0,0}(x)=\frac{b(x)}{C(1 ; x)}$,

2) $\varphi_{0, e_{p}, 0}(x)$ pour $p=1, \cdots, l$ sont arbitraires,

3) $\varphi_{0,0,1}(x) \equiv 0$,

et par (2.4), (2.7), (2.9) nous voyons que

4) $\varphi_{i, j, k}(x)(i+|j|+k \geqq 2)$ sont déterminés de manière unique par l'ensemble $\left\{\varphi_{0, e_{1}, 0}(x), \cdots, \varphi_{0, e_{l}, 0}(x)\right\}$ et sont holomorphes dans un même disque centré à l'origine de $\boldsymbol{C}^{n}$.

Nous avons ainsi obtenu une famille de solutions formelles de la forme (2.5). Comme le champ de vecteurs $T$ satisfait à une condition de Poincarè, le théorème 1 nous dit que la solution formelle est convergente au voisinage de l'origine $\left(t_{0}=0, t_{1}=0, \cdots, t_{l}=0, t_{l+1}=0\right)$. Ce qui prouve la proposition 1 .

Si $w\left(t_{0}, t_{1}, \cdots, t_{l}, t_{l+1}, x\right)$ est une solution de ( $\left.E_{2}^{\prime}\right)$ donnée par la proposition 1 , alors

$$
u(t, x)=w\left(t, t^{\rho_{1}(x)}, \cdots, t^{\rho_{l}(x)}, t \log t, x\right)
$$

est une famille de solutions de $\left(E_{2}\right)$ appartenant à $\widetilde{\mathcal{O}}_{+}$. Ce qui prouve la partie existence du théorème 2 .

Pour prouver l'unicité de la solution de $\left(\mathrm{E}_{2}\right)$ de la forme (2.2) il suffit de montrer que la solution formelle $\hat{u}(t, x)$ de $\left(\mathrm{E}_{2}\right)$ de la forme

$$
\hat{u}(t, x)=\sum_{i+|j|+k \geq 1} \varphi_{i, j, k}(x) t^{i}\left(t^{\rho_{1}(x)}\right)^{j_{1}} \cdots\left(t^{\rho_{l}(x)}\right)^{j_{l}}(t \log t)^{k}
$$

déterminée uniquement par la donnée des $\left\{\varphi_{0, j, 0}(x) ;|j|=1\right\}$.

Introduisons la condition:

$$
\left\{\begin{array}{l}
i+j_{1} \rho_{1}(x)+\cdots+j_{l} \rho_{l}(x) \neq p+q_{1} \rho_{1}(x)+\cdots+q_{l} \rho_{l}(x) \\
\text { dans } C\{x\} \text { pour tout }(i, j),(p, q) \in N \times N^{l} \\
\text { satisfaisant à }(i, j) \neq(p, q) .
\end{array}\right.
$$

Si la condition (2.11) est satisfaite, toute partie finie de l'ensemble de fonctions

$$
\left\{t^{i+j_{1} \rho_{1}(x)+\cdots+j_{l} \rho_{l}(x)}(t \log t)^{k} ; i+|j|+k \geqq 1\right\}
$$

est fonctionnellement indépendante: dans ce cas il est aisé de voir que les coéfficients $\varphi_{i, j, k}(x)$ figurant dans l'expression (2.10) de la solution formelle $\hat{\imath}(t, x)$ sont déterminés de manière unique par les fonctions $\left\{\varphi_{0, j, 0}(x) ;|j|=1\right\}$. 
Lorsque la condition (2.11) n'est pas satisfaite, prenons des fonctions $\lambda_{p}(x)$ $\in C_{r}\{x\} \quad(p=1,2, \cdots)$ et $\nu_{q}(x) \in C_{r}\{x\} \quad(q=1,2, \cdots, d)$ telles que

$$
\begin{cases}\lambda_{p}(x) \neq \nu_{q}(x) & \text { dans } C_{r}\{x\} \text { pour tout } p, q, \\ \lambda_{p_{1}}(x) \neq \lambda_{p_{2}}(x) & \text { dans } C_{r}\{x\} \text { si } p_{1} \neq p_{2}, \\ \nu_{q_{1}}(x) \neq \nu_{q_{2}}(x) & \text { dans } C_{r}\{x\} \text { si } q_{1} \neq q_{2},\end{cases}
$$

et tel que de plus comme parties de $\boldsymbol{C}_{r}\{x\}$ nous ayons

$$
\begin{aligned}
& \{1\} \cup\left\{i+j_{1} \rho_{1}(x)+\cdots+j_{l} \rho_{l}(x) ; i+|j| \geqq 2\right\} \\
& \quad=\left\{\lambda_{p}(x) ; p=1,2, \cdots\right\}, \\
& \left\{i+j_{1} \rho_{1}(x)+\cdots+j_{l} \rho_{l}(x) ; i+|j| \geqq 1\right\} \\
& \quad=\left\{\lambda_{p}(x) ; p=1,2, \cdots\right\} \cup\left\{\nu_{1}(x), \cdots, \nu_{d}(x)\right\} .
\end{aligned}
$$

La solution formelle se réecrit sous la forme

$$
\begin{aligned}
& \widehat{\imath}(t, x)=\sum_{p=1}^{l} \varphi_{0, e_{p}, 0}(x) t^{{ }^{\prime}{ }^{\prime}{ }^{(x)}}+\sum_{p=1}^{\infty} \sum_{k=0}^{\infty} \phi_{p, k}^{\lambda}(x) t^{\lambda{ }^{\prime}(x)}(t \log t)^{k} \\
& +\sum_{q=1}^{d} \sum_{k=1}^{\infty} \phi_{q, k}^{\nu}(x) t^{\nu} q^{(x)}(t \log t)^{k}+\sum_{k=1}^{\infty} \phi_{k}(x)(t \log t)^{k} .
\end{aligned}
$$

D'après la condition (2.4) par (2.12) nous obtenons:

$$
\begin{cases}C\left(\lambda_{p}(x)+k ; x\right) \neq 0 & \text { dans } D_{r} \text { pour } p \geqq 1 \text { et } k \geqq 0, \\ C\left(\nu_{q}(x)+k ; x\right) \neq 0 & \text { dans } D_{r} \text { pour } q=1, \cdots, d \text { et } k \geqq 1, \\ C(k ; x) \neq 0 & \text { dans } D_{r} \text { pour } k \geqq 1 .\end{cases}
$$

Donc, si nous prenons en compte le fait que $C\left(\rho_{p}(x) ; x\right) \equiv 0$ pour $p=1, \cdots, l$, par un calcul facile nous voyons que les coéfficients $\phi_{p, k}^{\lambda}(x), \phi_{q, k}^{\nu}(x)$ et $\phi_{k}(x)$ sont déterminés uniquement par $\varphi_{0, e_{1}, 0}(x), \cdots, \varphi_{0, e_{l}, 0}(x)$.

Nous avons ainsi obtenu l'unicité d'une solution formelle de la forme (2.2).

Nous obtenons également:

Corollaire 2. Soit $1 \leqq l \leqq m$. Supposons :

i) $\rho_{1}(x), \cdots, \rho_{l}(x)$ holomorphes au voisinage de $x=0$;

ii) $\operatorname{Re} \rho_{1}(0)>0, \cdots, \operatorname{Re} \rho_{l}(0)>0$;

iii) $C(1 ; 0) \neq 0$;

iv) $C\left(i+j_{1} \rho_{1}(0)+\cdots+j_{l} \rho_{l}(0)+k ; 0\right) \neq 0$ pour tout

$(i, j, k) \in \boldsymbol{N} \times \boldsymbol{N}^{l} \times \boldsymbol{N}$ satisfaisant $\grave{a} i+|j|+k \geqq 2$.

Alors, si $\left(\mathrm{E}_{2}\right)$ admet une solution formelle de la forme

$$
\hat{\imath}(t, x)=\sum_{i+\mid j_{1}+k \geqq 1} \varphi_{i, j, k}(x) t^{i}\left(t^{\rho_{1}(x)}\right)^{j_{1}} \cdots\left(t^{\rho_{l}(x)}\right)^{\jmath^{l}}(t \log t)^{k}
$$


où les coéfficients $\varphi_{i, j, k}(x)$ sont holomorphes dans un même disque centré à l'origine de $\boldsymbol{C}^{n}$, alors $\hat{u}(t, x)$ est convergente dans $\widetilde{\mathcal{O}}_{+}$.

Preuve. Comme nous avons unicité de la solution formelle, $\hat{\imath}(t, x)$ coincide avec la solution donnée par le théorème 2 ; elle est donc convergente dans $\widetilde{\mathcal{O}}_{+}$.

\section{§ 3. Solutions Singulières: Cas Général}

Comme généralisation des résultats du $\$ 2$, nous construisons dans cette section une famille de solutions appartenant à $\widetilde{O}_{+}$d'équations aux dérivées partielles singulières non linéaires de la forme:

$$
\begin{aligned}
\left(\left(t \frac{\partial}{\partial t}\right)^{m}\right. & \left.+c_{m-1}(x)\left(t \frac{\partial}{\partial t}\right)^{m-1}+\cdots+c_{0}(x)\right) u \\
= & t b(x)+G_{2}(x)\left(t,\left\{\left(t \frac{\partial}{\partial t}\right)^{j}\left(\frac{\partial}{\partial x}\right)^{\alpha} u\right\}_{j+\mid \cap_{1} \leqq m}\right),
\end{aligned}
$$

les notations et données étant les mêmes que dans le $\$ 2$.

La différence entre les équations $\left(\mathrm{E}_{2}\right)$ et $\left(\mathrm{E}_{3}\right)$ est la suivante:

-dans $\left(\mathrm{E}_{2}\right), Z_{j, \alpha}$ correspond à

$$
\left(t \frac{\partial}{\partial t}\right)^{j}\left(t \frac{\partial}{\partial x}\right)^{\alpha}=\left(t \frac{\partial}{\partial t}\right)^{j} t^{i \alpha_{1}}\left(\frac{\partial}{\partial x}\right)^{\alpha},
$$

-dans $\left(\mathrm{E}_{3}\right), Z_{j, \alpha}$ correspond à

$$
\left(t \frac{\partial}{\partial t}\right)^{j}\left(\frac{\partial}{\partial x}\right)^{\alpha}
$$

Il est clair qu'une équation du type $\left(\mathrm{E}_{2}\right)$ est un cas particulier d'une équation du type $\left(\mathrm{E}_{3}\right)$.

Comme dans le $\$ 2$ désignons par $\rho_{1}(x), \cdots, \rho_{m}(x)$ les exposants caractéristiques c'est à dire les racines du polynôme:

$$
C(\rho ; x)=\rho^{m}+\sum_{j<m} c_{j}(x) \rho^{j} .
$$

Nous avons:

Théorème 3. Soit $1 \leqq l \leqq m$. Supposons :

i) $\rho_{1}(x), \cdots, \rho_{l}(x)$ holomorphes au voisinage de $x=0$;

ii) $\operatorname{Re} \rho_{1}(0)>0, \cdots, \operatorname{Re} \rho_{l}(0)>0$;

iii) $C(1 ; 0) \neq 0$;

iv) $C\left(i+j_{1} \rho_{1}(0)+\cdots+j_{l} \rho_{l}(0) ; 0\right) \neq 0$ pour tout $(i, j) \in \boldsymbol{N} \times \boldsymbol{N}^{l}$ vérifiant $i+|j| \geqq 2$.

Alors l'équation $\left(\mathrm{E}_{3}\right)$ a une famille de solutions appartenant $\grave{a} \widetilde{O}_{+}$de la forme

$$
\sum_{i \geqq 1} u_{\imath}(x) t^{i}+\sum_{\substack{i+2 m|j| \geq k+2 m \\|j| \geq 1}} \varphi_{i, j, k}(x) t^{i+j_{1} \rho_{1}(x)+\cdots+j_{l} \rho_{l}(x)}(\log t)^{k},
$$


où tous les coéfficients $\varphi_{i, j, k}(x)$ sont holomorphes dans un même disque centré à l'origine de $C^{n}$; de plus tonte solution de $\left(\mathrm{E}_{3}\right)$ est déterminée de manière unique par la donnée des coéfficients $\left\{\varphi_{0, j, 0}(x) ;|j|=1\right\}$ qui sont arbitraires. Si nous prenons $\varphi_{0, j, 0}(x) \equiv 0$ pour tout $j$ tel que $|j|=1$, la solution (3.1) se réduit à l'unique solution holomorphe de l'équation $\left(\mathrm{E}_{3}\right)$.

Preuve. Nous commençons par construire une famille de solutions formelles de la forme (3.1).

Comme dans le (2.4), nous prenons un $r>0$ tel que $b(x) \in C_{r}\{x\}$ et

$$
\begin{cases}1) & C(1 ; x) \neq 0 \text { dans } D_{r} ; \\ \text { 2) } & C\left(i+j_{1} \rho_{1}(x)+\cdots+j_{l} \rho_{l}(x) ; x\right) \bullet_{r}^{\circ} 0 \text { dans } D_{r} \\ & \text { pour tout }(i, j) \in \boldsymbol{N} \times \boldsymbol{N}^{l} \text { satisfaisant à } i+|j| \geqq 2 .\end{cases}
$$

Pour tout $(i, 0) \in N^{*} \times N^{l}$ nous désignons par $\mathscr{F}_{r}(i, 0)$ l'ensemble des fonctions $f(t, x)$ de la forme

$$
f(t, x)=\phi(x) t^{i}, \quad \phi(x) \in C_{r}\{x\} .
$$

Pour tout $(i, j) \in \boldsymbol{N} \times\left(\boldsymbol{N}^{l} 、\{0\}\right)$ nous désignons par $\mathscr{F}_{r}(i, j)$ l'ensemble des fonctions $g(t, x)$ de la forme

$$
g(t, x)=\sum_{k=0}^{i+2 m(|j|-1)} \phi_{k}(x) t^{i+\jmath_{1} \rho_{1}(x)+\cdots+j_{l} \rho_{l}(x)}(\log t)^{k}
$$

où pour tout $k, \phi_{k}(x) \in C_{r}\{x\}$.

En posant

$$
\begin{cases}w_{l, 0}=u_{\imath}(x) t^{\ell} & \text { pour } i \geqq 1, \\ w_{0, e_{p}}=\varphi_{0, e_{p}, 0}(x) t^{\rho_{p}(x)} & \text { pour } p=1, \cdots, l, \\ w_{\imath, j}=\sum_{k=0}^{i+2 m(|j|-1)} \varphi_{l, j, k}(x) t^{i+j_{1} \rho_{1}(x)+\cdots+j_{l} \rho_{l}(x)}(\log t)^{k} & \text { pour } i+|j| \geqq 2 \text { et }|j| \geqq 1,\end{cases}
$$

la solution formelle $\hat{u}(t, x)$ de la forme (3.1) prend la forme

$$
\hat{u}(t, x)=\sum_{i+|j| \geqq 1} w_{i, j}(t, x)
$$

où pour tout $(i, j), w_{\imath, j} \in \mathscr{F}_{r}(i, j)$.

Il est facile de voir que la classe de fonctions $\mathscr{I}_{r}(i, j)(i+|j| \geqq 1)$ possède pour tout couple $(i, j)$ et $(p, q)$ appartenant à $N \times N^{l} \backslash\{(0,0)\}$ les propriétés suivantes :
1) $\mathscr{F}_{r}(i, j) \times \mathscr{F}_{r}(p, q) \subset \mathscr{F}_{r}(i+p, j+q)$;
2) $\left(t \frac{\partial}{\partial t}\right) \mathscr{F}_{r}(i, j) \subset \mathscr{F}_{r}(i, j)$; 
3) $\left(\frac{\partial}{\partial x}\right)^{\alpha} \mathscr{F}_{r}(i, j) \times\left(\frac{\partial}{\partial x}\right)^{\beta} \mathscr{F}_{r}(p, q) \subset \mathscr{F}_{r}(i+p, j+q)$, pour $|\alpha| \leqq m$ et $|\beta| \leqq m$. En regardant (3.4) nous voyons que l'équation $\left(\mathrm{E}_{3}\right)$ se déompose en les formules récurrentes :

$$
\left\{\begin{array}{l}
C\left(t \frac{\partial}{\partial t} ; x\right) w_{1,0}=b(x) t, \\
C\left(t \frac{\partial}{\partial t} ; x\right) w_{0, e_{p}}=0 \quad \text { pour } p=1, \cdots, l, \\
C\left(t \frac{\partial}{\partial t} ; x\right) w_{i, j}=F_{i, j}\left(w_{p, q} ;(p, q) \in A_{\imath, \jmath}\right) \quad \text { pour } i+|j| \geqq 2,
\end{array}\right.
$$

où

$$
A_{i, j}=\left\{(p, q) \in \boldsymbol{N} \times \boldsymbol{N}^{l} ; p \leqq i, q_{1} \leqq j_{1}, \cdots, q_{l} \leqq j_{l} \text { et } 1 \leqq p+|q|<i+|j|\right\}
$$

et $F_{i, j}$ est une fonction appartenant à $\mathscr{T}_{r}(i, j)$ déterminée par $\left\{w_{p, q} ;(p, q) \in A_{i, j}\right\}$.

De plus, (3.3) nous donne

$$
\begin{aligned}
C\left(t \frac{\partial}{\partial t} ; x\right) w_{i, 0}= & C(i ; x) u_{i}(x) t^{2} \quad \text { pour } i \geqq 1, \\
C\left(t \frac{\partial}{\partial t} ; x\right) w_{0, e_{p}}= & C\left(\rho_{p}(x) ; x\right) \varphi_{0, e_{p}, 0}(x) t^{\rho_{p}(x)} \quad \text { pour } p=1, \cdots, l, \\
C\left(t \frac{\partial}{\partial t} ; x\right) w_{i, j}= & \sum_{k=0}^{i+2 m(|j|-1)}\left\{C\left(i+j_{1} \rho_{1}(x)+\cdots+j_{l} \rho_{l}(x) ; x\right) \varphi_{l, j, k}(x)\right. \\
& \left.+\sum_{h=1}^{m} C_{h}^{*}(i, j, k ; x) \varphi_{\imath, j, k+h}(x)\right\} t^{i+\jmath_{1} \rho_{1}(x)+\cdots+\jmath_{l} \rho_{l}(x)}(\log t)^{k} \\
& \text { pour } i+|j| \geqq 2 \text { et }|j| \geqq 1
\end{aligned}
$$

où par convention $\varphi_{\imath, j, k+h}(x) \equiv 0$ pour $k+h>i+2 m(|j|-1)$ et où

$$
\begin{aligned}
& C_{h}^{*}(i, j, k ; x) \\
& =\sum_{q=h}^{m}\left(\begin{array}{l}
q \\
h
\end{array}\right) c_{q}(x)\left(i+j_{1} \rho_{1}(x)+\cdots+j_{l} \rho_{l}(x)\right)^{q-h}(k+1)(k+2) \cdots(k+h)
\end{aligned}
$$

et $c_{m}(x) \equiv 1$.

Par conséquent, $(3.2)$ et le fait que $C\left(\rho_{p}(x) ; x\right) \equiv 0(p=1, \cdots, l)$ entrainent :

1) $u_{1}(x)=\frac{b(x)}{C(1 ; x)} \in C_{r}\{x\}$;

2) $\varphi_{0, e_{p}, 0}(x) \in C_{r}\{x\}(p=1, \cdots, l)$ sont arbitraires ;

3) $u_{i}(x) \in C_{r}\{x\} \quad(i \geqq 2)$ et $\varphi_{i, j, k}(x) \in C_{r}\{x\} \quad(i+|j| \geqq 2$ et $|j| \geqq 1)$

sont déterminés par $\varphi_{0, e_{1}, 0}(x), \cdots, \varphi_{0, e_{l}, 0}(x)$.

Nous avons ainsi obtenu une famille de solutions formelles de la forme (3.2) comme dans le $\S 2$. Pour compléter la démonstration il suffit de montrer la convergence de cette solution formelle. 
Admettons pour le moment:

Lemme 1. Soit $1 \leqq l \leqq m$. Supposons vérifiées les hypothèses ii), iii) et iv) $d u$ théorème 3. Alors, il existe $N \in N^{*}$ tel que:

a) $\quad N \geqq 2 m+1$;

$\left.\mathrm{a}_{2}\right) \quad \operatorname{Re} \rho_{i}(0)>\frac{2 m}{N}$ pour tout $i=1, \cdots, l$;

a $) \quad C\left(\frac{2 m+1}{N} ; 0\right) \neq 0 ;$

$\left.\mathrm{a}_{4}\right) \quad C\left(\frac{i-2 m|\jmath|+k+2 m}{N}+j_{1} \rho_{1}(0)+\cdots+j_{l} \rho_{l}(0) ; 0\right) \neq 0$

pour tout $(i, j, k) \in \boldsymbol{N} \times \boldsymbol{N}^{\imath} \times \boldsymbol{N}$ vérifiant $i+|\jmath|+k \geqq 2$.

Démontrons maintenant la convergence de la solution formelle

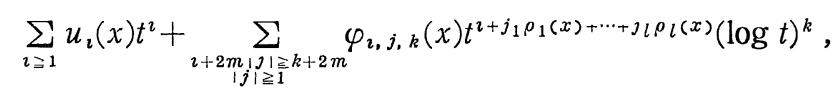

où les coéfficients $u_{i}(x)$ et $\varphi_{\imath, v_{k}}(x)$ sont tous holomorphes dans un même disque centré à l'origine de $\boldsymbol{C}^{n}$.

Soit $N$ un entier positif vérifiant les conditions du lemme 1 . Posons dans l'équation $\left(\mathrm{E}_{3}\right)$

$$
w(t, x)=t^{-2 m} u\left(t^{N}, x\right) ;
$$

il vient pour $w(t, x)$ l'équation :

$$
\begin{aligned}
\left(\left(\frac{1}{N} \theta_{2 m}\right)^{m}+\right. & \left.c_{m-1}(x)\left(\frac{1}{N} \theta_{2 m}\right)^{m-1}+\cdots+c_{0}(x)\right) w \\
= & t^{N-2 m} b(x)+\sum_{p+|q| \geqq 2} g_{p, q}(x) t^{N p-2 m+2 m|q|-L(q)} \\
& \times \operatorname{II}_{J+|a| \leqq m}\left\{\left(\frac{1}{N} \theta_{2 m-|a|}\right)^{j}\left(t \frac{\partial}{\partial x}\right)^{a} w\right\}^{q_{j, a}},
\end{aligned}
$$

où

$$
\theta_{s}=t \frac{\partial}{\partial t}+s \quad \text { et } \quad L(q)=\sum_{j+|\alpha| \leqq m}|\alpha| q_{j, a} .
$$

Comme $N \geqq 2 m+1$ et $p+|q| \geqq 2$, nous avons

$$
\begin{aligned}
N p-2 m+2 m|q|-L(q) & \geqq(2 m+1) p-2 m+2 m|q|-m|q| \\
& =(m+1) p+m(p+|q|-2) \\
& \geqq(m+1) p \geqq 0
\end{aligned}
$$

et 


$$
\begin{aligned}
(N p-2 m+2 m|q|-L(q))+|q| & \geqq(m+1) p+i q \mid \\
& \geqq p+|q| \geqq 2 .
\end{aligned}
$$

Ce qui montre que l'équation $\left(E_{3}^{*}\right)$ est un cas particulier d'équations $\left(E_{2}\right)$ que nous avons étudiées dans le $\$ 2$.

Désignons par $\lambda_{1}(x), \cdots, \lambda_{m}(x)$ les racines du polynôme:

$$
C^{*}(\lambda ; x)=\left(\frac{\lambda+2 m}{N}\right)^{m}+\sum_{j<m} c_{j}(x)\left(\frac{\lambda+2 m}{N}\right)^{j} .
$$

Nous avons:

1) $\lambda_{i}(x)=N \rho_{i}(x)-2 m$ pour tout $i=1, \cdots, m$;

2) $C *(\lambda ; x)=C\left(\frac{\lambda+2 m}{N} ; x\right)$.

La condition 1) et le lemme 1 entrainent que

i) $\lambda_{1}(x), \cdots, \lambda_{l}(x)$ sont holomorphes au voisinage de $x=0$;

ii) $\operatorname{Re} \lambda_{1}(0)>0, \cdots, \operatorname{Re} \lambda_{l}(0)>0$;

iii) $C^{*}(1 ; 0) \neq 0$;

iv) $C^{*}\left(i+j_{1} \lambda_{1}(0)+\cdots+j_{l} \lambda_{l}(0)+k ; 0\right) \neq 0$ pour tout $(i, j, k) \in \boldsymbol{N} \times \boldsymbol{N}^{l} \times \boldsymbol{N}$ vérifiant $i+|j|+k \geqq 2$.

Ce qui signifie que l'équation $\left(\mathrm{E}_{3}^{*}\right)$ vérifie les hypothèses du corollaire $2 \mathrm{du}$ $\S 2$.

La relation (3.6) transforme la solution formelle (3.5) en la série formelle

$$
\begin{aligned}
\sum_{i \geqq 1} u_{\imath}(x) t^{N i-2 m}+ & \sum_{\substack{t+2 m|j| \geq k k+2 m \\
|j| \geq 1}} N^{k} \varphi_{i, j, k}(x) t^{N i+2 m|\jmath|-k-2 m} \\
& \times\left(t^{\lambda_{1}(x)}\right)^{j_{1}} \cdots\left(t^{\lambda_{l}(x)}\right)^{\jmath^{l}}(t \log l)^{k}
\end{aligned}
$$

qui est un cas particulier de (2.13). Comme $\hat{\imath}(t, x)$ est une solution formelle de $\left(\mathrm{E}_{3}\right), w(t, x)$ est une solution formelle de $\left(\mathrm{E}_{3}^{*}\right)$.

Le corollaire 2 du $\S 2$ entraine la convergence de la solution formelle $w(t, x)$ dans $\widetilde{\mathcal{O}}_{+}$ce qui implique la convergence de $\hat{u}(t, x)$ dans $\widetilde{\mathcal{O}}_{+}$.

Le théorème 3 est donc démontré.

Démonstration du lemme 1. Notons

et

$$
J_{+}=\left\{i \in\{1, \cdots, m\} ; \operatorname{Re} \rho_{i}(0)>0\right\}
$$

$$
a=\min _{i \in J_{+}} \operatorname{Re} \rho_{i}(0) .
$$

Il est évident que $\{1, \cdots, l\} \in J_{+}$. Prenons maintenant $N \in N^{*}$ tel que

$$
N \geqq \max \left\{2 m+1, \frac{4 m}{a}, \frac{2 m+2}{a}\right\} .
$$

La condition $a_{1}$ ) est alors trivialement satisfaite. Les inégalités 


$$
\operatorname{Re} \rho_{l}(0)-\frac{2 m}{N} \geqq a-2 m\left(\frac{a}{4 m}\right)=\frac{a}{2}>0, \quad i=1, \cdots, l
$$

entraine la condition $a_{2}$ ). En remarquant que

$$
C\left(\frac{2 m+1}{N} ; 0\right)=\prod_{i=1}^{m}\left(\frac{2 m+1}{N}-\rho_{\imath}(0)\right)
$$

et que

$$
0<\frac{2 m+1}{N} \leqq(2 m+1)\left(\frac{a}{2 m+2}\right)<a \leqq \operatorname{Re} \rho_{i}(0), \quad i \in J_{+},
$$

nous obtenons la condition $a_{3}$ ).

Posons

$$
A=\max _{1 \equiv \imath \leq m} \operatorname{Re} \rho_{\imath}(0)
$$

el prenons un entier $M$ tel que

$$
M \geqq \max \left\{2, \frac{2 A}{a}\right\} .
$$

Alors, si $N$ satisfait (3.8) et si $(i, j, k) \in \boldsymbol{N} \times \boldsymbol{N}^{l} \times \boldsymbol{N}$ vérifie $|j|>M$, (3.9) nous donne:

$$
\begin{aligned}
& \operatorname{Re}\left\{\frac{i-2 m|j|+k+2 m}{N}+j_{1} \rho_{1}(0)+\cdots+j_{l} \rho_{l}(0)\right\} \\
& =\frac{i+k+2 m}{N}+\jmath_{1}\left(\operatorname{Re} \rho_{1}(0)-\frac{2 m}{N}\right)+\cdots+j_{l}\left(\operatorname{Re} \rho_{l}(0)-\frac{2 m}{N}\right) \\
& \quad \geqq \frac{2 m}{N}+\frac{a}{2}|j| \\
& >\frac{a}{2} M \geqq \frac{a}{2} \frac{2 A}{a}=A \geqq \operatorname{Re} \rho_{\imath}(0)
\end{aligned}
$$

pour tout $i=1, \cdots, m$. Ceci signifie que pour un choix de $N$ satisfaisant à (3.8) la condition $\left.\mathrm{a}_{1}\right)$ est satisfaite pour tout $(i, j, k) \in \boldsymbol{N} \times \boldsymbol{N}^{\iota} \times \boldsymbol{N}$ vérifiant $|j|$ $>M$.

Donc pour terminer la démonstration du lemme 1 , il suffit de choisir un $N$ vérifiant (3.8) et la condition:

$$
\left\{\begin{array}{l}
\frac{i-2 m|j|+k+2 m}{N} \neq \rho_{p}(0)-j_{1} \rho_{1}(0)-\cdots-j_{l} \rho_{l}(0) \\
\text { pour tout } p=1, \cdots, m \text { et tout }(i, j, k) \in N \times N^{l} \times N \\
\text { vérifiant } i+|j|+k \geqq 2 \text { et }|j| \leqq M .
\end{array}\right.
$$

Faisons l'étude de cette condition.

Pour $h=0,1, \cdots, M$, notons 


$$
S_{h}=\left\{\rho_{p}(0)-j_{1} \rho_{1}(0)-\cdots-j_{l} \rho_{l}(0) ; p=1, \cdots, m \text { et }|j|=h\right\} .
$$

Les conditions iii), iv) du lemme 1 nous donnent

$$
S_{h} \cap\{1,2, \cdots\}=\varnothing \quad \text { pour } h=0,1, \cdots, M .
$$

Il est facile de voir que la condition (3.10) est équivalente à

$$
\begin{cases}\frac{i+k+2 m}{N} \notin S_{0} & \text { pour } i+k \geqq 2, \\ \frac{i+k}{N} \notin S_{1} & \text { pour } i+k \geqq 1, \\ \frac{i-2 m h+k+2 m}{N} \notin S_{h} & \text { pour } i+k \geqq 0 \text { et } h=2, \cdots, M .\end{cases}
$$

De plus, comme $0 \notin S_{h}$ pour $h=2, \cdots, M$ (par iv) du lemme 1) il est aisé de voir que la condition (3.12) se réduit aux conditions :
$\left.\mathrm{b}_{1}\right) \frac{i+k+2 m}{N} \notin S_{0} \cap \boldsymbol{Q}_{+}$pour $i+k \geqq 2$,
$\left.\mathrm{b}_{2}\right) \frac{i+k}{N} \notin S_{1} \cap \boldsymbol{Q}_{+}$pour $\imath+k \geqq 1$,
$\left.\mathrm{b}_{3}\right) \quad \frac{i-2 m h+k+2 m}{N} \notin S_{h} \cap \boldsymbol{Q}_{+}$pour $i+k \geqq 0$ et $h=2, \cdots, M$,
$\left.\mathrm{b}_{4}\right) \quad \frac{i-2 m h+k+2 m}{N} \notin S_{h} \cap \boldsymbol{Q}_{-}$pour $i+k \geqq 0$ et $h=2, \cdots, M$,

où $\boldsymbol{Q}_{+}$[resp. $\left.\boldsymbol{Q}_{-}\right]$désignent l'ensemble des nombres rationnels strictement positifs [resp. strictement négatifs].

Posons

$$
\left(\bigcup_{h=0}^{M} S_{h}\right) \cap \boldsymbol{Q}_{+}=\left\{\alpha_{1}, \alpha_{2}, \cdots, \alpha_{K}\right\} .
$$

La condition (3.11) nous dit que pour tout $i=1,2, \cdots, K, \alpha_{\imath} \oplus\{1,2, \cdots\}$ donc $\alpha_{\imath}$ peut s'écrire

$$
\alpha_{\imath}=\frac{c_{\imath}}{d_{\imath}}
$$

pour des entiers non nuls $c_{i}$ et $d_{i}$ satisfaisant $c_{i} \geqq 1$ et $d_{i} \geqq 2$ et de plus premiers entre eux. Si $N \in N^{*}$ est premier avec tous les $d_{i}$ pour $i=1, \cdots, K$, alors pour tout $i, \alpha_{i} \oplus(\boldsymbol{Z} / N)$ et nous avons $\left.\left.\mathrm{b}_{1}\right), \mathrm{b}_{2}\right)$ et $\left.\mathrm{b}_{3}\right)$.

Posons maintenant

$$
S^{*}=\left(\bigcup_{h=2}^{M} S_{h}\right) \cap Q_{-} .
$$

Lorsque $S^{*}=\varnothing$, la condition $\mathrm{b}_{4}$ ) est triviale. Lorsque $S^{*} \neq \varnothing$, introduisons

$$
B=\min \left\{|\beta| ; \beta \in S^{*}\right\} \text {. }
$$


Si $N$ vérifie

$$
N>\frac{2 m M-2 m}{B}
$$

nous avons

$$
\frac{i-2 m h+k+2 m}{N} \geqq \frac{-(2 m M-2 m)}{N}>-B \geqq \beta
$$

pour tout $\beta \in S^{*}$ et donc la condition $\mathrm{b}_{4}$ ).

En conclusion, si on prend un entier $N$ qui vérifie (3.8), (3.13) et qui soit premier avec tous les entiers $d_{\imath}$ pour $i=1, \cdots, K$, toutes les conditions énoncées dans le lemme 1 seront vérifiées.

\section{$\S 4$. Analyse Asymptotique}

Dans ce paragraphe, nous rappelons la théorie asymptotique développée dans Tahara [14] pour les équations aux dérivées partielles linéaires et nous l'appliquons à quelques équations non linéaires.

Pour $a \in \boldsymbol{R}$ et toute fonction $u(t, x)$ nous écrivons

$$
\left.u(t, x)=O\left(t^{a} ; \check{\mathcal{O}}_{+}\right) \quad \text { (quand } t \rightarrow 0\right),
$$

lorsque nous avons $t^{-a} u(t, x) \in \widetilde{\mathcal{O}}_{+}$.

Tout d'abord nous étudions l'équation differentielle ordinaire suivante:

$$
\left(\left(t \frac{\partial}{\partial t}\right)^{m}+c_{m-1}(x)\left(t \frac{\partial}{\partial t}\right)^{m-1}+\cdots+c_{0}(x)\right) u=f,
$$

où $m \in N^{*}$, et $c,(x)(0 \leqq j \leqq m-1)$ sont des fonctions holomorphes définies dans un polydisque centré à l'origine de $C^{n}$.

Désignons par $\rho_{1}(x), \cdots, \rho_{m}(x)$ les racines du polynôme:

$$
C(\rho ; x)=\rho^{m}+\sum_{j<m} c_{\jmath}(x) \rho^{\jmath} .
$$

L'équation (4.1) peut alors s'écrire sous la forme

$$
\left(t \frac{\partial}{\partial t}-\rho_{1}(x)\right) \cdots\left(t \frac{\partial}{\partial t}-\rho_{m}(x)\right) u=f .
$$

La résolution explicite de (4.2) nous donne aisément:

Lemme 2. (1) Si $\int(t, x)=O\left(t^{a} ; \widetilde{\mathcal{O}}_{+}\right)$(quand $\left.t \rightarrow 0\right)$ pour un $a \in \boldsymbol{R}$ salisfaisant $\grave{a}$

$$
a<\min _{1 \leqq i \leqq m} \operatorname{Re} \rho_{i}(0),
$$

alors toute solution $u(t, x)$ de (4.1) vérifie

$$
u(t, x)=O\left(t^{a} ; \widetilde{\mathcal{O}}_{+}\right) \quad(\text { quand } t \rightarrow 0) .
$$


(2) Si $f(t, x)=O\left(t^{a} ; \widetilde{\mathcal{O}}_{+}\right)$(quand $\left.t \rightarrow 0\right)$ pour un $a \in \boldsymbol{R}$ satisfaisant $\grave{a}$

$$
a>\max _{1 \leq i \leq m} \operatorname{Re} \rho_{i}(0),
$$

alors l'équalion (4.1) a une solution unique $u(t, x)$ vérifiant

$$
u(t, x)=O\left(t^{a} ; \widetilde{\mathcal{O}}_{+}\right) \quad(\text { quand } t \rightarrow 0) .
$$

(3) Si $f(t, x) \equiv 0$ et si les nombres $\rho_{1}(0), \cdots, \rho_{m}(0)$ sont distincts, la solution générale de (4.1) est de la forme

$$
u(t, x)=\sum_{i=1}^{m} \varphi_{i}(x) t^{\rho_{\imath}(x)},
$$

où pour tout $i=1, \cdots, m$, les $\varphi_{\imath}(x) \in \boldsymbol{C}\{x\}$ sont arbitraires.

(4) Si $f(t, x)=O\left(t^{a} ; \widetilde{\mathcal{O}}_{+}\right)$(quand $t \rightarrow 0$ ) pour un $a \in \boldsymbol{R}$ satisfaisant $\grave{a}$

$$
a>\max _{1 \leqq i \leqq m} \operatorname{Re} \rho_{i}(0)
$$

et si les nombres $\rho_{1}(0), \cdots, \rho_{m}(0)$ sont distincts, alors toute solution de (4.1) est de la forme

$$
u(t, x)=\sum_{i=1}^{m} \varphi_{\iota}(x) t^{\rho_{\imath}(x)}+O\left(t^{a} ; \widetilde{\mathcal{O}}_{+}\right) \quad(\text { quand } t \rightarrow 0)
$$

pour des $\varphi_{i}(x) \in C\{x\} \quad(i=1, \cdots, m)$.

Nous allons donner maintenant une variante du lemme 2. Soient $I_{1}, I_{2}, I_{3}$ des sous ensembles de $\{1,2, \cdots, m\}$ satisfaisant aux conditions suivantes:

1) $I_{1} \cup I_{2} \cup I_{3}=\{1,2, \cdots, m\}$;

2) $I_{2} \neq \varnothing$;

3) $I_{i} \cap I_{j}=\varnothing$ si $i \neq j$;

4) $\max _{i \in I p} \operatorname{Re} \rho_{i}(0)<\min _{i \in I_{p+1}} \operatorname{Re} \rho_{\iota}(0)$ pour $p=1,2$.

Lorsque $I_{1} \neq \varnothing$ et $I_{3} \neq \varnothing$, nous poserons

$$
\begin{array}{ll}
M_{p}=\max _{i \in I p} \operatorname{Re} \rho_{i}(0) & \text { pour } p=1,2, \\
m_{p}=\min _{i \in I p} \operatorname{Re} \rho_{i}(0) & \text { pour } p=2,3 .
\end{array}
$$

Enfin si $I_{1}=\varnothing$ nous posons $M_{1}=-\infty$ et si $I_{3}=\varnothing$ nous posons $m_{3}=+\infty$.

Alors comme conséquence du lemme 2 nous obtenons:

Corollaire 3. Soient $a \in \boldsymbol{R}, b \in \boldsymbol{R}, u(t, x)$ et $f(t, x)$ deux fonctions. Supposons que:

i) $a<b$;

ii) $u(t, x)=O\left(t^{a} ; \widetilde{\widetilde{O}}_{+}\right)$(quand $\left.t \rightarrow 0\right)$;

iii) $f(t, x)=O\left(t^{b} ; \widetilde{\mathscr{O}}_{+}\right)($quand $t \rightarrow 0)$; 
iv) $u(t, x)$ et $f(t, x)$ satisfont $\grave{a}(4.1)$.

Alors:

(1) Si $M_{1}<a<b<m_{2}$, nous avons $u(t, x)=O\left(t^{b} ; \widetilde{\mathcal{O}}_{+}\right)$(quand $t \rightarrow 0$ ).

(2) Si $M_{2}<a<b<m_{3}$, nous avons $u(t, x)=O\left(t^{b} ; \widetilde{\mathcal{O}}_{+}\right.$) (quand $t \rightarrow 0$ ).

(3) Si $M_{1}<a<m_{2}$ et $M_{2}<b<m_{3}$ et si les $\rho_{i}(0)$ pour $i \in I_{2}$ sont distincts, nous avons

$$
u(t, x)=\sum_{i \in I_{2}} \varphi_{i}(x) t^{\rho i(x)}+O\left(t^{b} ; \widetilde{\mathcal{O}}_{+}\right) \quad(\text { quand } t \rightarrow 0)
$$

pour des $\varphi_{i}(x) \in C\{x\} \quad\left(i \in I_{2}\right)$.

Remarque. Si $I_{1}=\varnothing$, la conclusion (1) du lemme 2 entraine le résultat (1). Si $I_{3}=\varnothing$, la conclusion (2) du lemme 2 entraine le résultat (2). Si $I_{1}=I_{3}=\varnothing$, la conclusion (4) du lemme 2 entraine le résultat (3).

Démonstration dans le cas général. Posons

$$
C_{p}=\prod_{\imath \in I}\left(t \frac{\partial}{\partial t}-\rho_{i}(x)\right), \quad p=1,2,3 .
$$

$C_{p}$ est un opérateur différentiel à coéfficients holomorphes au voisinage de l'origine de $\boldsymbol{C}^{n}$ et l'équation (4.1) s'écrit:

$$
C_{1} C_{2} C_{3} u=C_{1} C_{3} C_{2} u=\int \text {. }
$$

Si $M_{1}<a<b<m_{2}$ posons $w=C_{2} C_{3} u$. Alors, $w=O\left(t^{a} ; \widetilde{\mathcal{O}}_{+}\right)$(quand $t \rightarrow 0$ ) et $C_{1} w=f=O\left(t^{b} ; \widetilde{O}_{+}\right)$(quand $t \rightarrow 0$ ). Donc d'après (2) du lemme 2 nous avons $w=$ $O\left(t^{b} ; \widetilde{\mathcal{O}}_{+}\right)$(quand $\left.t \rightarrow 0\right)$. Par conséquent, en appliquant (1) du lemme 2 à l'équation $C_{2} C_{3} u=w$ nous obtenons $u=O\left(t^{b} ; \widetilde{\mathscr{O}}_{+}\right)$(quand $t \rightarrow 0$ ) ce qui est la conclusion (1).

Si $M_{2}<a<b<m_{3}$, en posant $w=C_{3} u$ et par le même type d'arguments nous obtenons la conclution (2).

Si $M_{1}<a<m_{2}$ et $M_{2}<b<m_{3}$ nous posons $w_{1}=C_{3} C_{2} u$ et $w_{2}=C_{2} u$. Alors, $w_{p}=O\left(t^{a} ; \widetilde{\mathcal{O}}_{+}\right.$) (quand $t \rightarrow 0$ ) pour $p=1,2, C_{1} w_{1}=f$ et $C_{3} w_{2}=w_{1}$. En appliquant la conclusion (2) du lemme 2 à l'équation $C_{1} w_{1}=f$ nous obtenons $w_{1}=O\left(t^{b} ; \widetilde{\mathcal{O}}_{+}\right)$ (quand $t \rightarrow 0$ ); par suite, en appliquant la conclusion (1) du lemme 2 à l'équation $C_{3} w_{2}=w_{1}$ nous obtenons $w_{2}=O\left(t^{b} ; \widetilde{\mathcal{O}}_{+}\right.$) (quand $t \rightarrow 0$ ). Donc si les $\rho_{i}(0)$ pour $i \in I_{2}$ sont distincts, en appliquant la conclusion (4) du lemme 2 à l'équation $C_{2} u=w_{2}$ nous obtenons le résultat (3) du colloraire 3 .

Le corollaire 3 est donc bien une conséquence du lemme 2 .

Soit

$$
R[]: \widetilde{\mathcal{O}}_{+} \longrightarrow \widetilde{\mathcal{O}}_{+}
$$

un opérateur de $\widetilde{\mathcal{O}}_{+}$dans $\widetilde{\mathcal{O}}_{+}$. Considérons l'équation: 


$$
\left(\left(t \frac{\partial}{\partial t}\right)^{m}+c_{m-1}(x)\left(t \frac{\partial}{\partial t}\right)^{m-1}+\cdots+c_{0}(x)\right) u=R[u] .
$$

Soit $s>0$; imposons la condition suivante à $R$ :

$$
\left\{\begin{array}{l}
\text { Si } u(t, x)=O\left(t^{a} ; \widetilde{\mathcal{O}}_{+}\right) \text {(quand } t \rightarrow 0 \text { ) pour un } a>0, \\
\text { alors } R[u]=O\left(t^{b} ; \widetilde{\mathcal{O}}_{+}\right) \text {(quand } t \rightarrow 0 \text { ) pour tout } b<\min \{2 a, a+s\} .
\end{array}\right.
$$

Alors nous avons:

Lemme 3. Soient $I_{1}, I_{2}, I_{3}, M_{1}, M_{2}, m_{2}$ et $m_{3}$ comme dans le corollaire 3. Soit $s>0$ et supposons que l'opérateur $R[]: \widetilde{\mathcal{O}}_{+} \rightarrow \widetilde{\mathcal{O}}_{+}$satisfait à la condition $\left(\mathrm{B}_{s}\right)$. Supposons aussi que $u(t, x) \in \widetilde{\mathcal{O}}_{+}$soit une solution de (4.3). Alors:

(1) Si $u(t, x)=O\left(t^{a} ; \widetilde{\mathcal{O}}_{+}\right)$(quand $\left.t \rightarrow 0\right)$ pour un $a>\max \left\{0, M_{1}\right\}$, nous avons $u(t, x)=O\left(t^{b} ; \widetilde{\mathcal{O}}_{+}\right)$(quand $\left.t \rightarrow 0\right)$ pour tout $b<m_{2}$.

(2) Si $u(t, x)=O\left(t^{a} ; \widetilde{\mathcal{O}}_{+}\right)$(quand $t \rightarrow 0$ ) pour un $a>\max \left\{0, M_{2}\right\}$, nous avons $u(t, x)=O\left(t^{b} ; \widetilde{\mathcal{O}}_{+}\right)$(quand $\left.t \rightarrow 0\right)$ pour tout $b<m_{3}$.

(3) Si $u(t, x)=O\left(t^{a} ; \widetilde{\mathcal{O}}_{+}\right)$(quand $t \rightarrow 0$ ) pour un $a>0$ satisfaisant $\grave{a}$

$$
M_{1}<a<m_{2} \leqq M_{2}<\min \{2 a, a+s\}
$$

et si $\rho_{\imath}(0), i \in I_{2}$, sont distincts, nous avons

$$
u(t, x)=\sum_{i \in I_{2}} \varphi_{2}(x) t^{\rho_{\imath}(x)}+O\left(t^{b} ; \widetilde{\mathcal{O}}_{+}\right) \quad(\text { quand } t \rightarrow 0)
$$

pour des $\varphi_{i}(x) \in C\{x\} \quad\left(i \in I_{2}\right)$ et un nombre $b>M_{2}$.

Démonstration. Nous démontrons (1). Supposons que $u(t, x)=\left(t^{a} ; \widetilde{\mathcal{O}}_{+}\right)$ (quand $t \rightarrow 0$ ) pour un $a>\max \left\{0, M_{1}\right\}$, et soit $b>0$ tel que $a<b<m_{2}$. Prenons une suite $a_{1}, a_{2}, \cdots, a_{N}$ vérifiant:

1) $a=a_{1}<a_{2}<\cdots<a_{N}=b$;

2) $a_{i+1}<\min \left\{2 a_{i}, a_{i}+s\right\}$ pour tout $i=1,2, \cdots, N-1$.

Posons $f(t, x)=R[u](t, x) \in \widetilde{\mathcal{O}}_{+}$. Comme nous avons $u(t, x)=O\left(t^{a_{1}} ; \widetilde{\mathcal{O}}_{+}\right)$(quand $t \rightarrow 0$ ), en utillisant $\left(\mathrm{B}_{s}\right)$ et la condition $a_{2}<\min \left\{2 a_{1}, a_{1}+s\right\}$ nous avons $f(t, x)$ $=O\left(t^{a_{2}} ; \widetilde{\mathcal{O}}_{+}\right.$) (quand $t \rightarrow 0$ ) et d'après la conclusion (1) du corollaire 3 nous avons $u(t, x)=O\left(t^{a_{2}} ; \widetilde{\mathcal{O}}_{+}\right)$(quand $\left.t \rightarrow 0\right)$ ce qui signifie que $f(t, x)=O\left(t^{a_{3}} ; \widetilde{\mathcal{O}}_{+}\right)$(quand $t \rightarrow 0$ ) comme $a_{3}<\min \left\{2 a_{2}, a_{2}+s\right\}$. En utilisant à nouveau la conclusion (1) du colloraire 3 nous obtenons $u(t, x)=O\left(t^{a_{3}} ; \widetilde{\mathcal{O}}_{+}\right.$) (quand $\left.t \rightarrow 0\right)$. Et par récurrence nous obtenons ainsi $u(t, x)=O\left(t^{a} ; \widetilde{\mathcal{O}}_{+}\right)$(quand $t \rightarrow 0$ ) ce qui prouve la conclusion (1) du lemme 3.

La conclusion (2) du lemme 3 se démontre de la même manière.

Démontrons (3). Supposons que $u(t, x)=\left(t^{a} ; \widetilde{\mathcal{O}}_{+}\right.$) (quand $t \rightarrow 0$ ) pour un $a>0$ satisfaisant à

$$
M_{1}<a<m_{2} \leqq M_{2}<\min \{2 a, a+s\} .
$$

Prenons $b \in \boldsymbol{R}$ tel que 


$$
M_{2}<b<\min \left\{2 a, a+s, m_{3}\right\} .
$$

Posons $f(t, x)=R[u](t, x) \in \widetilde{\mathscr{O}}_{+}:$par $\left(\mathrm{B}_{s}\right)$ nous avons $f(t, x)=O\left(t^{b} ; \widetilde{\mathcal{O}}_{+}\right)$(quand $t \rightarrow 0)$; par suite en appliquant la conclusion (3) du corollaire 3 nous obtenons (4.5) pour des $\varphi_{i}(x) \in C\{x\} \quad\left(i \in I_{2}\right)$. La partie (3) du lemme 3 se trouve ainsi démontrée.

Nous allons appliquer les résultats du lemme 3 à certaines équations aux dérivées partielles non linéaires.

Soient :

$-a_{j, \alpha}(t, x) \in \widetilde{\mathcal{O}}_{+}$pour $j+|\alpha| \leqq m$; par définition il existe $s>0$ tel que

$$
\left.a_{j, \alpha}(t, x)=O\left(t^{s} ; \widetilde{\mathcal{O}}_{+}\right) \text {(quand } t \rightarrow 0\right) \quad \text { pour tout } j+|\alpha| \leqq m .
$$

$-\mathscr{R}(\boldsymbol{C} \backslash\{0\})$ le revêtement universel de $\boldsymbol{C} \backslash\{0\}$;

$-S(\varepsilon(s))=\{t \in \mathscr{R}(\boldsymbol{C} \backslash\{0\}) ; 0<|t|<\varepsilon(\arg t)\}$ où $\varepsilon(s)$ est une fonction définie sur

$\boldsymbol{R}$ continue et strictement positive;

$-D_{r}=\left\{x \in \boldsymbol{C}^{n} ;\left|x_{i}\right| \leqq r, i=1, \cdots, n\right\}$.

Posons

$$
Z=\left\{Z_{j, \alpha}\right\}_{j+1 a \mid \leqq m}, \quad Z_{j, \alpha} \in C ;
$$

soit $G_{2}(t, x)(Z)$ une fonction holomorphe en les varables $(t, x, Z)$ définie dans $S(\varepsilon(s)) \times D_{r} \times\left\{Z ;\left|Z_{j, \alpha}\right| \leqq r\right\}$ et ayant la forme suivante :

$$
G_{2}(t, x)(Z)=\sum_{|q| \geqq 2} g_{q}(t, x) Z^{q}
$$

où

$$
\begin{aligned}
q & =\left\{q_{j, \alpha}\right\}_{j+|\alpha| \leqq m}, \quad q_{j, n} \in N, \\
|q| & =\sum_{j+|\alpha| \leqq m} q_{j, a}, \\
Z^{q} & =\prod_{j+|\alpha| \leqq m}\left(Z_{j, a}\right)^{q_{j, n}}
\end{aligned}
$$

et $g_{q}(t, x) \quad(|q| \geqq 2)$ sont des fonctions holomorphes définies dans $S(\varepsilon(s)) \times D_{r}$. De plus supposons que pour tout $\theta>0$ et toute partie compacte $K \times L$ de $D_{r} \times$ $\left\{Z ;\left|Z_{j, \alpha}\right| \leqq r\right\}$ nous ayons

$$
\max _{(x, Z) \in K \times L}\left|G_{2}(t, x)(Z)\right|=O(1)
$$

quand $t \rightarrow 0$ dans $S_{\theta}$.

Considérons l'équation suivante: 
$\left(\mathrm{E}_{1}\right)$

$$
\begin{aligned}
\left(\left(t \frac{\partial}{\partial t}\right)^{m}\right. & \left.+c_{m-1}(x)\left(t \frac{\partial}{\partial t}\right)^{m-1}+\cdots+c_{0}(x)\right) u \\
= & \sum_{j+|\alpha| \leqq m} a_{j, \kappa}(t, x)\left(t \frac{\partial}{\partial t}\right)^{j}\left(\frac{\partial}{\partial x}\right)^{a} u \\
& +G_{2}(t, x)\left(\left\{\left(t \frac{\partial}{\partial t}\right)^{j}\left(\frac{\partial}{\partial x}\right)^{\alpha} u\right\}_{j+|a| \leqslant m}\right) .
\end{aligned}
$$

Si nous posons

$$
\begin{aligned}
R[u]= & \sum_{j+|\alpha| \leqq m} a_{j, \alpha}(t, x)\left(t \frac{\partial}{\partial t}\right)^{j}\left(\frac{\partial}{\partial x}\right)^{a} u \\
& +G_{2}(t, x)\left(\left\{\left(t \frac{\partial}{\partial t}\right)^{j}\left(\frac{\partial}{\partial x}\right)^{\alpha} u\right\}_{j+|a| \leqq m}\right),
\end{aligned}
$$

les conditions (4.6) et (4.7) ainsi que le fait que $R$ satisfait à la condition $\left(\mathrm{B}_{s}\right)$ nous permet d'appliquer le lemme 3 . Nous obtenons ainsi:

Proposition 2. Soient $I_{1}, I_{2}, I_{3}, M_{1}, M_{2}, m_{2}$ et $m_{3}$ comme dans le corollaire 3. Supposons que $u(t, x) \in \widetilde{\mathcal{O}}_{+}$soit une solution de $\left(\mathrm{E}_{4}\right)$. Alors:

(1) Si $u(t, x)=O\left(t^{a} ; \widetilde{\mathcal{O}}_{+}\right)$(quand $t \rightarrow 0$ ) pour un $a>\max \left\{0, M_{1}\right\}$, nous avons $u(t, x)=O\left(t^{b} ; \widetilde{\mathcal{O}}_{+}\right)$(quand $\left.t \rightarrow 0\right)$ pour tout $b<m_{2}$.

(2) Si $u(t, x)=O\left(t^{a} ; \widetilde{\mathcal{O}}_{+}\right)$(quand $t \rightarrow 0$ ) pour un $a>\max \left\{0, M_{2}\right\}$, nous avons $u(t, x)=O\left(t^{b} ; \widetilde{O}_{+}\right)($quand $t \rightarrow 0)$ pour tout $b<m_{3}$.

(3) Si $u(t, x)=O\left(t^{a} ; \widetilde{\mathcal{O}}_{+}\right)$(quand $\left.t \rightarrow 0\right)$ pour un $a>0$ satisfaisant $\grave{a}$

$$
M_{1}<a<m_{2} \leqq M_{2}<\min \{2 a, a+s\}
$$

ct si $\rho_{i}(0), i \in I_{2}$, sont distincts, alors nous avons

$$
u(t, x)=\sum_{i \in I_{2}} \varphi_{i}(x) t^{\rho_{2}(x)}+O\left(t^{b} ; \widetilde{\mathcal{O}}_{+}\right) \quad(\text { quand } t \rightarrow 0)
$$

pour des $\varphi_{i}(x) \in C\{x\} \quad\left(i \in I_{2}\right)$ et un nombre $b>M_{2}$.

Pour terminer discutons l'unicité de la solution de $\left(\mathrm{E}_{4}\right)$. Soit $u(t, x) \in \widetilde{\mathcal{O}}_{+}$ une solution de $\left(\mathrm{E}_{4}\right)$; supposons que $u(t, x)=O\left(t^{a} ; \widetilde{\mathcal{O}}_{+}\right.$) (quand $t \rightarrow 0$ ) pour un $a>0$ satisfaisant à

$$
a>\max _{1 \leqq i \leqq m} \operatorname{Re} \rho_{i}(0) .
$$

La conclusion (2) de la proposition 2 avec l'hypothèse que $I_{1}=I_{3}=\varnothing$ et $m_{3}=\infty$ nous donne:

$$
\left.u(t, x)=O\left(t^{\infty} ; \widetilde{\mathcal{O}}_{+}\right) \quad \text { (quand } t \rightarrow 0\right) ;
$$

c'est à dire que $u(t, x)=O\left(t^{b} ; \widetilde{\mathscr{O}}_{+}\right.$) (quand $t \rightarrow 0$ ) pour tout $b>0$.

Nous avons beaucoup plus: 
Proposition 3. Soit $u(t, x) \in \widetilde{\mathcal{U}}_{+}$une solution de $\left(\mathrm{E}_{4}\right)$ vérifiant $u(t, x)=$ $O\left(t^{a} ; \widetilde{\mathscr{O}}_{+}\right)$(quand $t \rightarrow 0$ ) pour un $a>0$ satisfaisant $\grave{a}$

$$
a>\max _{1 \leqq i \leqq m} \operatorname{Re} \rho_{i}(0) .
$$

Alors $u(t, x) \equiv 0$ dans $\widetilde{\mathcal{O}}_{+}$.

Démonstration. Posons

$$
b_{j, a}(t, x)=\int_{0}^{1} \frac{\partial G_{2}(t, x)}{\partial Z_{j, \alpha}}\left(\left\{\left(t \frac{\partial}{\partial t}\right)^{j}\left(\frac{\partial}{\partial x}\right)^{\alpha} u(t, x) \theta\right\}_{j+|\alpha| \leq m}\right) d \theta .
$$

Comme $a>0$, par (4.7) nous avons

$$
b_{j, a}(t, x)=O\left(t^{a} ; \tilde{\mathcal{O}}_{+}\right) \quad(\text { quand } t \rightarrow 0) .
$$

Soit $s>0$ comme dans (4.6), et $\rho>0$ tel que

$$
0<\rho<\min \left\{\frac{a}{m}, \frac{s}{m}\right\} .
$$

Posons

et

$$
w(t, x)=t^{-a} u(t, x) \in \widetilde{\mathcal{O}}_{+}
$$

$$
c_{j, \Omega}(t, x)=t^{-\rho_{|\alpha|}}\left(a_{j, \kappa}(t, x)+b_{j, \kappa}(t, x)\right) \in \widetilde{\mathcal{O}}_{+} .
$$

Il est alors aisé de voir que $u^{\prime}(t, x)$ est solution de l'équation aux dérivées partielles linéaires:

$$
C\left(t \frac{\partial}{\partial t}+a ; x\right) w=\sum_{j+|\alpha| \leqslant m} c_{j, \alpha}(t, x)\left(t \frac{\partial}{\partial t}-\rho|\alpha|+a\right)^{j}\left(t^{\rho} \frac{\partial}{\partial x}\right)^{a} w .
$$

De plus si $\lambda_{1}(x), \cdots, \lambda_{m}(x)$ désignent les racines du polynôme $C(\lambda+a ; x)$ la condition (4.9) entraine

$$
\operatorname{Re} \lambda_{i}(0)<0 \quad \text { pour tout } i=1, \cdots, m .
$$

Par suite en appliquant le théorème d'unicité de Tahara [13] ou BaouendiGoulaouic [1] à l'équation (4.10) nous obtenons $u(t, x) \equiv 0$ dans $\widetilde{\mathcal{O}}_{+}$.

\section{§. Fin de la Démonstration du Théorème Principal}

Dans ce paragraphe, nous appliquons les résultats des $\S 1$ à 4 aux équations aux dérivées partielles singulières non linéaires de la forme

$$
\left(t \frac{\partial}{\partial t}\right)^{m} u=F\left(t, x,\left\{\left(t \frac{\partial}{\partial t}\right)^{j}\left(\frac{\partial}{\partial x}\right)^{\alpha} u\right\}_{(j, a) \in I_{m}}\right)
$$

introduites dans notre introduction et nous complétons la démonstration du théorème principal.

Dans tout cette section nous supposons vérifiées les conditions $\left(A_{1}\right),\left(A_{2}\right)$ et 
$\left(\mathrm{A}_{3}\right)$ (voir l'introduction). Désignons par $\rho_{1}(x), \cdots, \rho_{m}(x)$ les fonctions de $x$ racines du polynôme:

$$
C(\rho ; x)=\rho^{m}-\sum_{j<m}\left(\frac{\partial F}{\partial Z_{j, 0}}(0, x, 0)\right) \rho^{\jmath} .
$$

Rappelons les notations :

$$
\begin{aligned}
& J_{+}=\left\{i \in\{1, \cdots, m\} ; \operatorname{Re} \rho_{i}(0)>0\right\} . \\
& \mu=\text { le cardinal de } J_{+} .
\end{aligned}
$$

Lorsque $\mu \geqq 1$ quitte à changer la numérotation nous supposons que :

$$
\begin{cases}\operatorname{Re} \rho_{i}(0)>0 & \text { pour } 1 \leqq i \leqq \mu, \\ \operatorname{Re} \rho_{i}(0) \leqq 0 & \text { pour } \mu+1 \leqq \imath \leqq m\end{cases}
$$

Les conditions $\left(A_{1}\right),\left(A_{2}\right)$ et $\left(A_{3}\right)$ entrainent que l'équation (E) s'écrit sous la forme

$$
\begin{aligned}
& \left(\left(t \frac{\partial}{\partial t}\right)^{m}+c_{m-1}(x)\left(t \frac{\partial}{\partial t}\right)^{m-1}+\cdots+c_{0}(x)\right) u \\
& =t b(x)+G_{2}(x)\left(t,\left\{\left(t \frac{\partial}{\partial t}\right)^{j}\left(\frac{\partial}{\partial x}\right)^{\alpha} u\right\}_{(j, a) \in I_{m}}\right)
\end{aligned}
$$

qui est un cas particulier d'équations du type $\left(\mathrm{E}_{1}\right)$ et $\left(\mathrm{E}_{3}\right)$. De plus nous avons

$$
c_{j}(x)=-\frac{\partial F}{\partial Z_{j, 0}}(0, x, 0), \quad j=0,1, \cdots, m-1 .
$$

Les résultats dans $\S 1$ à 4 nous donnent:

$\left(\mathrm{C}_{1}\right)$ (voir corollaire 1 ). $\quad \mathrm{Si} \rho_{i}(0) \notin N^{*}$ pour tout $i=1, \cdots, m$, l'équation (E) a une solution unique $u_{0}(t, x)\left(=u_{0}\right)$ holomorphe au voisinage de l'origine de $\boldsymbol{C}_{t} \times \boldsymbol{C}_{x}^{n}$ vèrifiant $u_{0}(0, x) \equiv 0$.

$\left(\mathrm{C}_{2}\right)$ (voir théorème 3 ). Supposons $\mu \geqq 1,(5.1)$ et les conditions suivantes:

i) $\rho_{1}(x), \cdots, \rho_{\mu}(x)$ sont holomorphes au voisinage de $x=0$;

ii) $C(1 ; 0) \neq 0$;

iii) $C\left(i+j_{1} \rho_{1}(0)+\cdots+j_{\mu} \rho_{\mu}(0) ; 0\right) \neq 0$ pour tout

$(i, j) \in \boldsymbol{N} \times \boldsymbol{N}^{\mu}$ satisfaisant à $i+|j| \geqq 2$.

Alors pour tout $\varphi_{1}(x), \cdots, \varphi_{\mu}(x) \in \boldsymbol{C}\{x\}$ l'équation (E) admet une solution unique $U\left(\varphi_{1}, \cdots, \varphi_{\mu}\right)(t, x)$ appartenant à $\widetilde{\mathcal{O}}_{+}$de la forme

$$
\begin{aligned}
& U\left(\varphi_{1}, \cdots, \varphi_{\mu}\right)=\sum_{i \geqq 1} u_{i}(x) t^{i}
\end{aligned}
$$

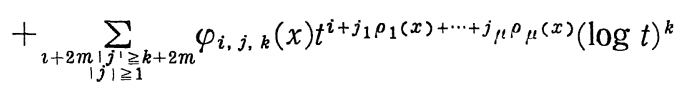

avec $\varphi_{0, e_{p}, 0}(x)=\varphi_{p}(x), p=1, \cdots, \mu$ où $e_{1}=(1,0, \cdots, 0), \cdots, e_{\mu}=(0, \cdots, 0,1)$. Il 
est immédiat de voir que $U(0, \cdots, 0)=u_{0}$ qui est la solution holomorphe unique donnée dans $\left(\mathrm{C}_{1}\right)$.

$\left(\mathrm{C}_{3}\right)$ (voir la discussion des $\S 2$ à 4 ). Soit $0 \leqq p<l \leqq \mu$. Supposons qu'il existe des nombres réels $s, a, b$ vérifiant les conditions suivantes:

1) $0<s<\min \left\{1, \min _{i \leqq p} \operatorname{Re} \rho_{i}(0)\right\}$;

2) $\max _{i \leqq p} \operatorname{Re} \rho_{i}(0)<a<\min _{p+1 \leqq i \leqq l} \operatorname{Re} \rho_{i}(0)$;

3) $\max _{p+1 \leqq i \leqq l} \operatorname{Re} \rho_{i}(0)<b<\min \{2 a, a+s\}$.

Alors sous les conditions données dans $\left(\mathrm{C}_{2}\right)$ nous avons

$$
\begin{aligned}
& U\left(\varphi_{1}, \cdots, \varphi_{l}, 0, \cdots, 0\right)-U\left(\varphi_{1}, \cdots, \varphi_{p}, 0, \cdots, 0\right)-\sum_{i=p+1}^{l} \varphi_{i}(x) t^{\rho_{i}(x)} \\
& \left.=O\left(t^{b} ; \widetilde{\mathcal{O}}_{+}\right) \quad \text { (quand } t \rightarrow 0\right) .
\end{aligned}
$$

Désignons par $\mathcal{S}_{+}$l'ensemble des solutions de (E) appartenant à $\widetilde{\mathcal{O}}_{+}$et retournons à la démonstration du théorème principal.

La condition $\left(\mathrm{C}_{1}\right)$ ci-dessus nous donne immédiatement la conclusion (I) du théorème principal. Les conditions $\left(\mathrm{C}_{1}\right)$ et $\left(\mathrm{C}_{2}\right)$ signifient que

$$
\mathcal{S}_{+} \supset\left\{\begin{array}{l}
\left\{u_{0}\right\}, \quad \text { quand } \mu=0, \\
\left\{U\left(\varphi_{1}, \cdots, \varphi_{\mu}\right) ;\left(\varphi_{1}, \cdots, \varphi_{\mu}\right) \in(C\{x\})^{\mu}\right\}, \quad \text { quand } \mu \geqq 1 .
\end{array}\right.
$$

Donc, pour compléter la démonstration du théorème principal il suffit de prouver:

Théorème 4. Supposons que $(\mathrm{E})$ satisfait aux conditions $\left(\mathrm{A}_{1}\right),\left(\mathrm{A}_{2}\right)$ et $\left(\mathrm{A}_{3}\right)$. Alors :

(1) Si $\mu=0$ c'est à dire que $\operatorname{Re} \rho_{i}(0) \leqq 0$ pour tout $i=1, \cdots, m, \mathcal{S}_{+}=\left\{u_{0}\right\}$.

(2) $S i \mu \geqq 1$ et si on a (5.1) ainsi que

i) $\rho_{i}(0) \neq \rho_{j}(0)$ pour $1 \leqq i \neq j \leqq \mu$;

ii) $C(1 ; 0) \neq 0$;

iii) $C\left(i+j_{1} \rho_{1}(0)+\cdots+j_{\mu} \rho_{\mu}(0) ; 0\right) \neq 0$ pour tout $(i, j) \in \boldsymbol{N} \times \boldsymbol{N}^{\mu}$ satisfaisant $\grave{a} i+|j| \geqq 2$;

toute solution $u \in \mathcal{S}_{+}$de (E) est de la forme

$$
u=U\left(\varphi_{1}, \cdots, \varphi_{l}\right)
$$

pour des $\varphi_{1}, \cdots, \varphi_{\mu}$ appartenant à $C\{x\}$ déterminés unıquement par $u$.

Démonstration. Soit $u \in \mathcal{S}_{+}$. Posons $w=u-u_{0}$. D'après la définition de $\widetilde{\mathcal{O}}_{+}$ nous avons pour un $a>0, w(t, x)=O\left(t^{a} ; \widetilde{\mathcal{O}}_{+}\right)$(quand $\left.t \rightarrow 0\right)$. Comme $u$ et $u_{0}$ sont des solutions de (E), $w$ est solution de 


$$
\begin{aligned}
\left(t \frac{\partial}{\partial t}\right)^{m} w= & F\left(t, x,\left\{\left(t \frac{\partial}{\partial t}\right)^{j}\left(\frac{\partial}{\partial x}\right)^{\alpha} w+\left(t \frac{\partial}{\partial t}\right)^{j}\left(\frac{\partial}{\partial x}\right)^{a} u_{0}(t, x)\right\}_{(j, \alpha) \in I_{m}}\right) \\
& -F\left(t, x,\left\{\left(t \frac{\partial}{\partial t}\right)^{j}\left(\frac{\partial}{\partial x}\right)^{\alpha} u_{0}(t, x)\right\}_{(j, \alpha) \in I_{m}}\right) .
\end{aligned}
$$

Les hypothèses $\left(A_{1}\right),\left(A_{2}\right)$ et $\left(A_{3}\right)$ entrainent que l'équation (5.3) pour $w$ est un cas particulier d'équation $\left(E_{4}\right)$. Par suite si nous supposons que $\operatorname{Re} \rho_{i}(0) \leqq 0$ pour tout $i=1, \cdots, m$ nous avons

$$
a>0 \geqq \max _{1 \leqq i \leqq m} \operatorname{Re} \rho_{i}(0)
$$

et la proposition 3 nous donne $w \equiv 0$. Ce qui prouve l'assertion (1) du théorème 4.

Pour démontrer (2) supposons donc avoir $\mu \geqq 1$, (5.1) et i), ii), iii). Nous pouvons supposer sans nuire à la généralité (en changeant éventuellement la numérotation) que

$$
\begin{aligned}
& 0<\operatorname{Re} \rho_{1}(0)=\cdots=\operatorname{Re} \rho_{i_{1}}(0) \\
& <\operatorname{Re} \rho_{i_{1}+1}(0)=\cdots=\operatorname{Re} \rho_{i_{2}}(0) \\
& \quad<\cdots<\operatorname{Re} \rho_{i_{N-1}+1}(0)=\cdots=\operatorname{Re} \rho_{i_{N}}(0)
\end{aligned}
$$

avec $0<i_{1}<i_{2}<\cdots<i_{N}=\mu$. Posons $i_{0}=0$ et

$$
m_{l}=\operatorname{Re} \rho_{i_{l-1}+1}(0)=\cdots=\operatorname{Re} \rho_{i_{l}}(0), \quad l=1, \cdots, N .
$$

Choisissons $s>0, a_{1}, \cdots, a_{N}, a_{N+1}=\infty$ et $b_{1}, \cdots, b_{N}$ tels que

1) $0<s<\min \left\{m_{1}, 1\right\}$;

2) $a_{l}<m_{l}<b_{l}<\min \left\{2 a_{l}, a_{l}+s, a_{l+1}\right\}, l=1, \cdots, N$.

Soit $u \in \mathcal{S}_{+}$fixé, posons $w_{1}=u-u_{0}$; comme $w_{1} \in \widetilde{\mathcal{O}}_{+}$nous avons pour un $a>0$, $w_{1}=O\left(t^{a} ; \widetilde{\mathcal{O}}_{+}\right)$(quand $\left.t \rightarrow 0\right)$. En appliquant la conclusion (1) de proposition 2 à $w_{1}$ qui est solution d'équation (5.3) qui est de la forme $\left(\mathrm{E}_{4}\right)$ nous obtenons $w_{1}=$ $O\left(t^{a_{1}} ; \widetilde{\mathcal{O}}_{+}\right)$(quand $t \rightarrow 0$ ) et la conclusion (3) de la même proposition nous donne

$$
w_{1}=\sum_{p=1}^{i_{1}} \varphi_{p}(x) t^{\rho_{p}(x)}+O\left(t^{b_{1}} ; \widetilde{\mathscr{O}}_{+}\right) \quad(\text { quand } t \rightarrow 0)
$$

pour des $\varphi_{1}(x), \cdots, \varphi_{i_{1}}(x) \in C\{x\}$.

Posons maintenant

$$
u_{1}=U\left(\varphi_{i}, \cdots, \varphi_{i_{1}}, 0, \cdots, 0\right) \quad \text { et } \quad w_{2}=u-u_{1}:
$$

$\operatorname{par}(5.4)$ et $\left(\mathrm{C}_{3}\right)$ nous avons 


$$
\begin{aligned}
w_{2}= & u_{0}-u_{1}+w_{1} \\
= & U(0, \cdots, 0)-U\left(\varphi_{1}, \cdots, \varphi_{i_{1}}, 0, \cdots, 0\right) \\
& +\sum_{p=1}^{i_{1}} \varphi_{p}(x) t^{\rho_{p}(x)}+O\left(t^{b_{1}} ; \widetilde{\mathcal{O}}_{+}\right) \\
= & O\left(t^{b_{1}} ; \widetilde{\mathcal{O}}_{+}\right) \quad(\text { quand } t \rightarrow 0) .
\end{aligned}
$$

De plus $w_{2}$ est solution de

$$
\begin{aligned}
\left(t \frac{\partial}{\partial t}\right)^{m} w_{2}= & F\left(t, x,\left\{\left(t \frac{\partial}{\partial t}\right)^{j}\left(\frac{\partial}{\partial x}\right)^{a} w_{2}+\left(t \frac{\partial}{\partial t}\right)^{j}\left(\frac{\partial}{\partial x}\right)^{a} u_{1}(t, x)\right\}_{(j, a) \in I_{m}}\right) \\
& -F\left(t, x,\left\{\left(t \frac{\partial}{\partial t}\right)^{j}\left(\frac{\partial}{\partial x}\right)^{a} u_{1}(t, x)\right\}_{(j, a) \in I_{m} n}\right)
\end{aligned}
$$

qui est une forme particulière de $\left(\mathrm{E}_{4}\right)$. Donc, par (2) de la proposition 2 nous avons $w_{2}=O\left(t^{a_{2}} ; \widetilde{\mathcal{O}}_{+}\right)$(quand $t \rightarrow 0$ ). En appliquant (3) de la même proposition nous obtenons

$$
\left.u_{2}=\sum_{p=i_{1}+1}^{i_{2}} \varphi_{p}(x) t^{\rho_{p}(x)}+O\left(t^{b_{2}} ; \widetilde{\mathcal{O}}_{+}\right) \quad \text { (quand } t \rightarrow 0\right)
$$

pour des $\varphi_{i_{1}+1}(x), \cdots, \varphi_{i_{2}}(x) \in C\{x\}$.

Introduisons maintenant

$$
u_{2}=U\left(\varphi_{1}, \cdots, \varphi_{i_{2}}, 0, \cdots, 0\right) \quad \text { et } \quad w_{3}=u-u_{2}:
$$

par (5.5) et $\left(\mathrm{C}_{3}\right)$ nous avons

$$
\begin{aligned}
w_{3}= & u_{1}-u_{2}+w_{2} \\
= & U\left(\varphi_{1}, \cdots, \varphi_{i_{1}}, 0, \cdots, 0\right)-U\left(\varphi_{1}, \cdots, \varphi_{i_{2}}, 0, \cdots, 0\right) \\
& +\sum_{p=i_{1}+1}^{\imath_{2}} \varphi_{p}(x) t^{\rho_{p}(x)}+O\left(t^{b_{2}} ; \widetilde{\mathcal{O}}_{+}\right) \\
= & \left.O\left(t^{b_{2}} ; \widetilde{\mathcal{O}}_{+}\right) \quad \text { (quand } t \rightarrow 0\right) .
\end{aligned}
$$

En répètant cet argument, nous obtenons des fonctions $\varphi_{i_{2^{+1}}}(x), \cdots, \varphi_{\mu}(x)$ $\in \boldsymbol{C}\{x\}$ telles que si nous posons

$$
u_{l}=U\left(\varphi_{1}, \cdots, \varphi_{i_{l}}, 0, \cdots, 0\right)
$$

pour $l \in\{3, \cdots, N\}$ et $w_{l+1}=u-u_{l}$ nous avons

$$
\left.w_{l+1}=O\left(t^{b_{l}} ; \widetilde{\Theta}_{+}\right) \quad \text { (quand } t \rightarrow 0\right) .
$$

En particulier pour $l=N, w_{N+1}$ vérifie

$$
\begin{aligned}
\left(t \frac{\partial}{\partial t}\right)^{m} w_{N+1}= & F\left(t, x,\left\{\left(t \frac{\partial}{\partial t}\right)^{j}\left(\frac{\partial}{\partial x}\right)^{a} w_{N+1}+\left(t \frac{\partial}{\partial t}\right)^{j}\left(\frac{\partial}{\partial x}\right)^{\alpha} u_{N}(t, x)\right\}_{(j, a) \in I_{m}}\right) \\
& -F\left(t, x,\left\{\left(t \frac{\partial}{\partial t}\right)^{j}\left(\frac{\partial}{\partial x}\right)^{\alpha} u_{N}(t, x)\right\}_{(j, a) \in I_{m}}\right)
\end{aligned}
$$


qui est une équation du type $\left(\mathrm{E}_{4}\right)$. Comme $w_{N+1}=O\left(t^{b} ; \widetilde{\mathcal{O}}_{+}\right)$(quand $t \rightarrow 0$ ) et que $b_{N}>m_{N}$ la proposition 3 entraine $w_{N+1} \equiv 0$ c'est à dire que

ce qui pouve (5.2).

$$
u=u_{N}=U\left(\varphi_{1}, \cdots, \varphi_{\mu}\right)
$$

Pour obtenir l'unicité il suffit de prouver que

$$
U\left(\varphi_{1}, \cdots, \varphi_{\mu}\right)=U\left(\psi_{1}, \cdots, \phi_{\mu}\right) \quad \text { dans } \widetilde{\mathcal{O}}_{+}
$$

entraine $\varphi_{i}(x)=\phi_{i}(x)$ dans $C\{x\}$ pour tout $i=1, \cdots, \mu$.

D'après (5.6) nous avons

$$
\begin{aligned}
0 & =U\left(\varphi_{1}, \cdots, \varphi_{\mu}\right)-U\left(\phi_{1}, \cdots, \phi_{\mu}\right) \\
& \left.=\sum_{p=1}^{i_{1}}\left(\varphi_{p}(x)-\psi_{p}(x)\right) t^{\rho_{p}(x)}+O\left(t^{b_{1}} ; \widetilde{\mathcal{O}}_{+}\right) \quad \text { (quand } t \rightarrow 0\right) .
\end{aligned}
$$

Comme $\operatorname{Re} \rho_{p}(0)<b_{1}$ pour $p=1, \cdots, i_{1}$ et les $\rho_{1}(0), \cdots, \rho_{\imath_{1}}(0)$ sont tous distincts, (5.7) nous donne

$$
\varphi_{p}(x)=\phi_{p}(x) \quad \text { dans } C\{x\} \text { pour tout } p=1, \cdots, i_{1} .
$$

Maintenant (5.6) et (5.8) entrainent facilement

$$
\begin{aligned}
0 & =U\left(\varphi_{1}, \cdots, \varphi_{\mu}\right)-U\left(\phi_{1}, \cdots, \phi_{\mu}\right) \\
& \left.=\sum_{p=i_{1}+1}^{i_{2}}\left(\varphi_{p}(x)-\phi_{p}(x)\right) t^{\rho_{p}(x)}+O\left(t^{b_{2}} ; \widetilde{\mathcal{O}}_{+}\right) \quad \text { (quand } t \rightarrow 0\right)
\end{aligned}
$$

ce qui donne

$$
\varphi_{p}(x)=\phi_{p}(x) \quad \text { dans } C\{x\} \text { pour tout } p=i_{1}+1, \cdots, i_{2} .
$$

Et par récurrence nous obtenons

$$
\varphi_{p}(x)=\phi_{p}(x) \quad \text { dans } C\{x\} \text { pour tout } p=1, \cdots, \mu
$$

ce qui termine la démonstration du théorème 4 et complète la démonstration du théorème principal de cet article.

\section{Bibliographie}

[1] Baouendi, M.S. et Goulaouic, G., Cauchy problem with characteristic initial hypersurface, Comm. Pure Appl. Math., 26 (1973), 455-475.

[2] Gérard, R., Une classe d'équations aux dérivées partielles non linéaires à singularité réguliere, Séminaire Vaillant, Propagation of singularities and differential operators, 53-71, Travaux en Cours, Hermann, 1985.

[3] - Une classe d'opérateurs singuliers non linéaires à singularité réguliere, Séminaire d'analyse P. Lelong--P. Dolbeault--H. Skoda, 146-162, Lecture Notes in Math., 1198, Springer, 1986.

[4] Gérard, R. et Tahara, H., Nonlinear singular first order partial differential equa- 
tions of Briot-Bouquet type, Proc. Japan Acad., 66-3 (1990), 72-74.

[5] Gérard, R. et Tahara, H., Holomorphic and singular solutions of nonlinear singular first order partial differential equations, Publ. RIMS, Kyoto Univ., 26 (1990), 9791000 .

[6] - Théorème du type Maillet pour une classe d'équations aux dérivées partielles analytiques singulières, C.R. Acad. des Sciences, Paris, 312 (1991), 499502 .

[7] - Maillet's type theorems for non linear singular partial differential equations, to appear in J. Math. Pures et Appl., Paris.

[8] — Maillet's type theorems for non linear singular partial differential equations without linear part, preprint of IRMA, Strasbourg.

[9] Ishii, T., On propagation of regular singularities for solutions of nonlinear partial differential equations, J. Fac. Sci. Univ. Tokyo Sec. IA, Math., 37 (1990), 377-424.

[10] Madi, N.S. et Yoshino, M., Uniqueness and solvability of nonlinear Fuchsian equations, Bull. Sc. Math., 2e série, 114 (1990), 41-60.

[11] Tahara, H., Fuchsian type equations and Fuchsian hyperbolic equations, Japan. J. Math., 5 (1979), 245-347.

[12] - Fundamental systems of analytic solutions of Fuchsian type partial differential equations, Funkcialaj Ekvacioj, 24 (1981), 135-140.

[13] ——, On a Volevic system of singular partial differential equations, J. Math. Soc. Japan, 34 (1982), 279-288.

[14] - Singular hyperbolic systems, V. Asymptotic expansions for Fuchsian hyperbolic partial differential equations, J. Math. Soc. Japan, 36 (1984), 449-473. 
